\title{
MAGNETOSPHERIC RESPONSE TO THE INTERACTION WITH THE SPORADIC SOLAR WIND DIAMAGNETIC STRUCTURE
}

\section{V.A. Parkhomov}

Baikal State University, Irkutsk, Russia, pekines_41@mail.ru

V.G. Eselevich

Institute of Solar-Terrestrial Physics SB RAS, Irkutsk,Russia,esel@iszf.irk.ru

M.V. Eselevich

Institute of Solar-Terrestrial Physics SB RAS, Irkutsk,Russia,mesel@isz.irk.ru

\section{A.V. Dmitriev}

Lomonosov Moscow State University, Skobeltsyn Institute of Nuclear Physics, Moscow,Russia,dalexav@mail.ru

\author{
A.V. Suvorova \\ Lomonosov Moscow State University, \\ Moscow, Russia, alla_suvorova@mail.ru
}

\section{S.Yu. Khomutov}

Institute of Cosmophysical Research and Radio Wave Propagation FEB RAS,

Paratunka,Russia, khomutov@ikir.ru

\section{B. Tsegmed}

Institute of Astronomy and Geophysics AS Mongolia, Ulaan-Baatar, Mongolia, tseg@iag.ac.mn

\section{T. Raita}

Sodankyla Geophysical Observatory, University of Oulu, Sodankyla,Finland, tero.raita@sgo.fi

\begin{abstract}
We report the results of a study on the movement of the solar wind diamagnetic structure (DS), which is a sequence of smaller-scale microDS being part of the May 18, 2013 coronal mass ejection, from a source on the Sun to Earth's surface. DS determined from the high negative correlation coefficient $(r=-0.9)$ between the IMF modulus $(B)$ and the SW density $(N)$ on the ACE and Wind satellites at the L1 point, on the THB and THC satellites $(r=-0.9)$ in nearEarth orbit, and on the THA satellite inside the magnetosphere is carried by the solar wind from the Sun to Earth's orbit, while maintaining its fine internal structure. Having a large size in the radial direction $(\approx 763$ $R_{\mathrm{E}}$, where $R_{\mathrm{E}}$ is the Earth radius), DS flows around the magnetosphere. At the same time, microDS of size $\leq 13$ $R_{\mathrm{E}}$ passes through the bow shock and magnetopause as a magnetized plasmoid in which the ion concentration increases from $10 \mathrm{~cm}^{-3}$ to $90 \mathrm{~cm}^{-3}$, and the velocity decreases as it moves toward the magnetotail. When a microDS passes through the magnetopause, a pulsed electric field of $\sim 400 \mathrm{mV} / \mathrm{m}$ is generated with subsequent oscillations with a period of $T \sim 200 \mathrm{~s}$ and an amplitude of $\sim 50 \mathrm{mV} / \mathrm{m}$. The electric field accelerates charged particles of the radiation belt and produces modulated fluxes of protons in an energy range $95-575 \mathrm{keV}$ on the day side and
\end{abstract}

electrons in $40-475 \mathrm{keV}$ and protons in $95-575 \mathrm{keV}$ on the night side. In the duskside magnetosphere (19-23 MLT), the substorm activation is observed in geomagnetic pulsations and auroras, but without a magnetic negative bay. In the post-midnight sector (01-05 MLT), a sawtooth substorm occurs without the growth phase and breakup with deep modulation of the ionospheric current and auroral absorption. The duration of all phenomena in the magnetosphere and on Earth is determined by the period of interaction between DS and the magnetosphere ( $4 \mathrm{hrs})$. To interpret the regularities of the magnetospheric response to the interaction with DS, we consider alternative models of the impulsive passage of DS from SW to the magnetosphere and the classical model of reconnection of IMF and the geomagnetic field.

Keywords: diamagnetic structure, magnetized plasmoid, impulsive passage to the magnetosphere, sawtooth substorm.

\section{INTRODUCTION}

Modern methods of space observations and a large amount of data on the state of the Sun, solar wind (SW), and Earth's magnetosphere make it possible to study the movement of agents of specific magnetospheric disturbances all the way from a source on the Sun to Earth. One of these agents may be a diamagnetic structure (DS) of the solar wind. The analysis carried out in [Eselevich, Eselevich, 2005, Parkhomov et al., 2018] has revealed that the antisunward extensions of higher brightness rays of the streamer belt (heliospheric plasma sheet) and streamer chains, recorded at Earth's orbit, are diamagnetic tubes or DS, which comprise the backbone of quasi-stationary slow SW. The DS interaction with
Earth's magnetosphere generates substorms of various types [Parkhomov et al., 2020]. DS has a fractal structure, i.e. it is embedded magnetic tubes (structures) whose diameter can decrease by almost two orders of magnitude [Eselevich, Eselevich, 2005]. That is why, at different time scales there are smaller DS within larger DS, which we conventionally call microDS.

Unlike quasi-stationary SW, sporadic SW, whose source on the Sun is coronal mass ejections (CMEs), in Earth's orbit is recorded as a sequence of a shock, shockheated plasma (sheath), and interplanetary coronal mass ejection (ICME) in the form of a magnetic cloud (MC) or in the form of a plasma ejection (ejecta) [Ermolaev et al., 2009]. Inside MC or ejecta, we can often see a bending 
thin magnetic rope with an increased plasma density, which arises due to a filament ejection from the solar surface (or eruptive prominence). This heliospheric process can be seen in full in Figure 1 in [Parkhomov et al., 2018].

The eruptive prominence is a sequence of microDS of sporadic SW. The microDS-Earth's magnetosphere collision can also cause a short-term (20-40 min) activation of magnetospheric processes, similar to the substorm one [Parkhomov et al., 2017].

This work is a sequel to the study on features of the interaction between the magnetosphere and the solar wind diamagnetic structures. We analyze the May 18, 2013 event: determine the solar source of sporadic SW DS, examine the appearance of DS in Earth's orbit as a sequence of microDS, and delve into the response of the magnetosphere to its interaction with microDS. This paper is the first to study microDS propagation into Earth's magnetosphere.

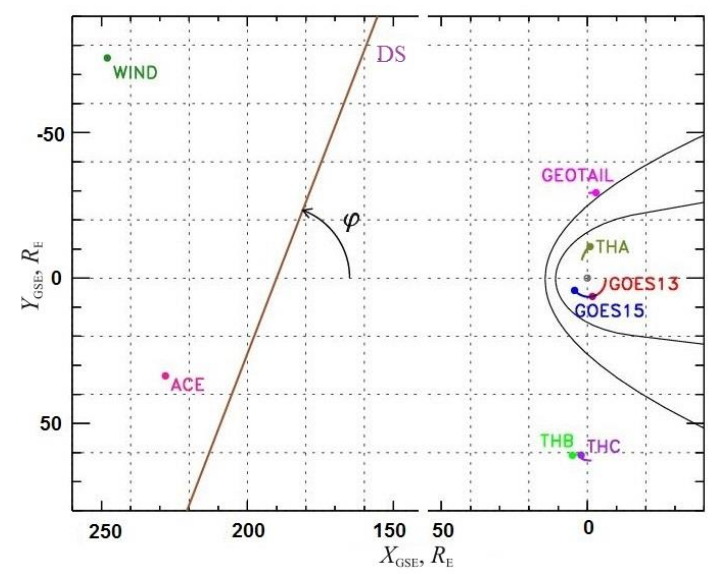

Figure 1. Orbits of the satellites whose data we use: the dot is the beginning of a satellite trajectory. The slant line indicates the orientation of the leading edge of DS

\section{DATA AND METHODS OF ANALYSIS}

To solve this problem, we have used the results of solar observations, published on the Website [https://cdaw.gsfc.nasa.gov/CME_list/catalog_description. $\mathrm{htm}$, one-minute values of SW and IMF parameters reduced to the position of near-Earth bow shock. We have applied observations from satellites ACE, Wind, and of THEMIS series (THA, THB, THC, THD, THE) [https://cdaweb.sci.gsfc.nasa.gov/cdaweb/ istp_public], geomagnetic indices, as well as GOES-13 and GOES-15 records of the geomagnetic field, proton and electron fluxes in the geostationary orbit [https:/satdat.ngdc.noaa.gov/sem/goes/ data/new_avg]. The satellite orbits are shown in Figure 1, and their coordinates for 00 UT on May 18, 2013 are listed in Table 1: $x_{\mathrm{GSE}}, y_{\mathrm{GSE}}, z_{\mathrm{GSE}}$ are coordinates of the satellites in the solar-ecliptic coordinate system, as well as modulus of the radius vector of $\mathrm{SC}$ position in Earth radii $\left(R_{\mathrm{E}}\right)$.

We have used ground-based geomagnetic observations (Table 2) made at observatories of the INTERMAGNET, IMAGE and CARISMA networks, as well as data on geomagnetic pulsations from the observatories Mondy, Magadan, and Paratunka, and on auroral absorption from the SGO meridional riometer network [https://www.sgo.fi/Data/Riometer/rioData.php].

\section{IDENTIFICATION OF DS SOURCE}

Consider a DS identified by a high negative correlation coefficient $(r=-0.91)$ between variations in the plasma density $N$ and the interplanetary magnetic field (IMF) modulus $B$, which was recorded in the Wind satellite orbit on May 18, 2013 at 00:45-04:00 UT (Figure 2).

Note that there is a shock before the DS at $\sim 00: 20$ UT (on May 18, 2013).

It is followed by a region of shock-heated plasma, recorded at 00:20-00:45 UT. In the classification of SW stream types according to the catalog [Ermolaev et al., 2009], this region is called sheath. It is followed by the ejecta region (00:45-04:00 UT), which includes the DS of interest, or, more precisely, a sequence of microDS of different scales. According to [Ermolaev et al., 2009], ejecta is one of the ICME manifestations, when a magnetic cloud is not detected. This happens if an ICME source on the Sun is located near the limb.

Let us try to identify the source of this ejecta (or DS) on the Sun. As the most probable source, we take CME

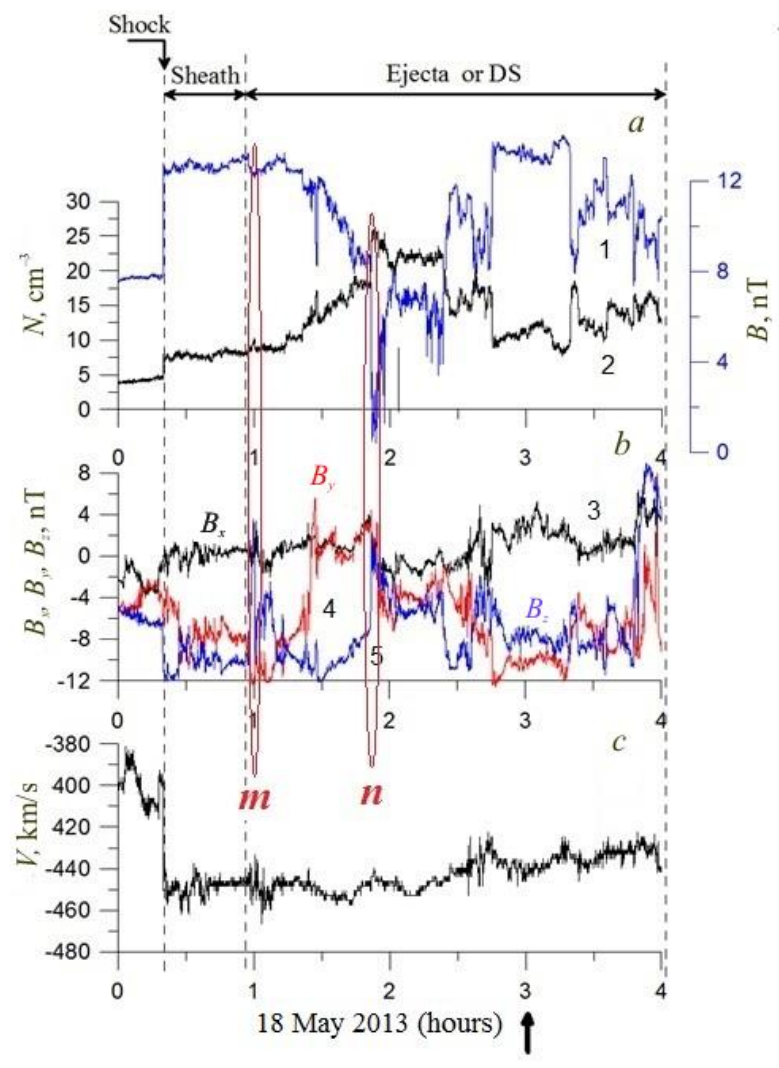

Figure 2. SW parameters according to the Wind satellite data for DS on May 18, 2013 at 00:00-04:00 UT with a time resolution of $3 \mathrm{~s}$ : IMF modulus $B(1), \mathrm{SW}$ plasma density $N$ (2), IMF components $B_{x}(3), B_{y}(4), B_{z}(5)$, SW speed $V(c)$. Vertical dashed lines mark the moments of the appearance of a shock, shock-heated plasma (sheath), and a structure ejected from the Sun (ejecta), which is actually a sequence of microDS of different scales. The vertical arrow at the bottom indicates the estimated arrival time of DS in Earth's orbit; $m, n$ are DS microstructures selected for the study 
Table 1

\begin{tabular}{|l|r|r|r|c|}
\hline Satellite & $x_{\mathrm{GSE}}, \mathrm{km}$ & \multicolumn{1}{|c|}{$y_{\mathrm{GSE}}, \mathrm{km}$} & $z_{\mathrm{GSE}}, \mathrm{km}$ & $\begin{array}{c}\text { Modulus of the } \\
\text { radius vector of } \\
\text { SC position, } R_{\mathrm{E}}\end{array}$ \\
\hline ACE & 1454660 & 214414 & 581098 & 259.874 \\
\hline Wind & 1581700 & -482550 & 116410 & 259.879 \\
\hline THA & 6369 & 68968 & 3165 & 10.8567 \\
\hline THB & 32550 & 388570 & -5733 & 61.4098 \\
\hline THC & 13504 & 387729 & -6364 & 61.1561 \\
\hline THD & 6368 & 7516 & 917 & 1.44143 \\
\hline THE & -12618 & 6363 & -1337 & 6.33477 \\
\hline Geotail & -18910 & -187310 & 21902 & 29.67 \\
\hline GOES-13 & -33187 & 22040 & 13631 & 6.61239 \\
\hline GOES-15 & 2802 & 42042 & 2866 & 6.61019 \\
\hline
\end{tabular}

Table 2

\begin{tabular}{|c|c|c|c|c|}
\hline No. & Station name & $\begin{array}{l}\text { Geographic } \\
\text { latitude, }{ }^{\circ}\end{array}$ & $\begin{array}{l}\text { Gographic } \\
\text { longitude, }\end{array}$ & $\begin{array}{l}\text { Magnetometer type, } \\
\text { sampling rate }\end{array}$ \\
\hline 1 & Abisko (ABI) & 68.4 & 18.8 & Riometer, $30 \mathrm{MHz}$ \\
\hline 2 & Chibougamau (CHBG) & 49.8 & 285.6 & All-sky camera \\
\hline 3 & Guam (GUA) & 70.2 & 15.8 & Fluxgate, $1 \mathrm{~min}$ \\
\hline 4 & Dawson (DAWS) & 64.0 & 220.8 & $\begin{array}{l}\text { ICM*, } 20 \mathrm{~Hz} ; \\
\text { Fluxgate, } 1 \mathrm{~Hz}\end{array}$ \\
\hline 5 & Fort Churchill (FCHU) & 58.7 & 265.9 & $\begin{array}{l}\text { ICM, } 20 \mathrm{~Hz} ; \\
\text { Fluxgate, } 1 \mathrm{~Hz}\end{array}$ \\
\hline 6 & Magadan (MGD) & 59.9 & 150.8 & $\begin{array}{l}\text { ICM, } 64 \mathrm{~Hz} \\
\text { Fluxgate, } 1 \mathrm{~Hz} \\
\end{array}$ \\
\hline 7 & Mondy (MND) & 52.1 & 104.4 & ICM, $64 \mathrm{~Hz}$ \\
\hline 8 & Pello (PEL) & 66.9 & 24.1 & Flux gate, $1 \mathrm{~Hz}$ \\
\hline 9 & Pevek (PBK) & 70.1 & 170.9 & Flux gate, 1 min \\
\hline 10 & Paratunka (PET) & 52.9 & 158.2 & $\begin{array}{l}\text { ICM, } 64 \mathrm{~Hz} ; \\
\text { Fluxgate, } 1 \mathrm{~Hz}\end{array}$ \\
\hline 11 & Pinava (PINA) & 50.2 & 263.9 & $\begin{array}{l}\text { ICM, } 20 \mathrm{~Hz} \\
\text { Fluxgate, } 1 \mathrm{~Hz}\end{array}$ \\
\hline 12 & Tamanrasset (TAM) & 22.8 & 5.5 & Fluxgate, $1 \mathrm{~min}$ \\
\hline 13 & Thief River Falls (THRF) & 48.0 & 263.6 & ICM, $20 \mathrm{~Hz}$ \\
\hline
\end{tabular}

that occurred on the east limb and was observed in white light in the field of view of the C2 LASCO coronagraph on May 15, 2013 at 01:48-02:24 UT. Sequence of its difference images is displayed in Figure 3, $a-c$. In Figure $3, a, b$, inside the white circle corresponding to the solar surface are difference images in a $193 \AA$ ultraviolet channel. Obviously, this $\mathrm{CME}$ is linked to a local brightness burst in the $193 \AA$ channel (a black line is drawn through it in Figure 3, $a, b$ ) and to an X-ray burst X1.2 (coordinates of the N12E64 flare) with a maximum at 01:40 UT (Figure 3,d). The mean velocity of this CME in the plane of the limb in region $2 R_{\odot}<$ $R<25 R_{\odot}\left(R_{\odot}\right.$ is the solar radius) $V_{0}$ limb $\approx 1370 \mathrm{~km} / \mathrm{s}$ [https://satdat.ngdc.noaa.gov/sem/goes/data/new_avg].

According to the results obtained by Schwenn et al. [2005], in the direction of the Sun-Earth line the average initial velocity of this CME region will be as fol- lows: $V_{0}{ }_{\mathrm{S}-\mathrm{E}} \approx V_{0} \mathrm{limb} / 1.8 \approx 761 \mathrm{~km} / \mathrm{s}$. Knowing the initial CME velocity in the direction to Earth near the Sun $V_{0 \text { S-E }}$, we can estimate the time of arrival of this CME region in Earth's orbit, as well as its velocity at Earth's orbit. According to [Eselevich, Eselevich, 2004], the average velocity $V_{\text {avS-E }}$ of this CME region from the Sun to a distance of $1 \mathrm{AU}$ is estimated by the relation

$$
V_{\text {av S-E }} \approx 3 / 4 V_{0 \text { S-E }} \text {, }
$$

and its velocity $V_{\mathrm{eS}-\mathrm{E}}$ at a distance of $1 \mathrm{AU}$ :

$$
V_{\text {e S-E }} \approx V_{0 \text { S-E }} / 2 \text {. }
$$

From Formulas (1) and (2), find $V_{\text {av }}=570 \mathrm{~km} / \mathrm{s}$, $V_{\mathrm{eS}-\mathrm{E}} \approx 380 \mathrm{~km} / \mathrm{s}$.

Estimate the time $\Delta T$ of movement of this CME region from the Sun to Earth:

$\Delta T \approx 215 R_{\odot} / V_{\text {av S-E }} \approx 1.5 \cdot 10^{8}[\mathrm{~km}] / 570[\mathrm{~km} / \mathrm{s}] \approx$ $\approx 73 \mathrm{hrs} \approx 3$ days $1 \mathrm{hr}$ 
The time of emergence of the CME under study on the Sun $t_{0} \approx 01: 48$ UT on May 15, 2013; time of arrival at a distance of $1 \mathrm{AU} t_{\mathrm{e}} \approx t_{0}+\Delta T \approx 03: 00 \mathrm{UT}$ on May 18, 2013 (marked with a vertical arrow in Figure 2 ). In this case, the estimated SW velocity at a distance of 1 AU $V_{\mathrm{eS}-\mathrm{E}} \approx 380 \mathrm{~km} / \mathrm{s}$ slightly differs from the recorded one of $\sim 440 \mathrm{~km} / \mathrm{s}$. Thus, the solar source of the DS of interest is the limb CME that occurred at $t_{0} \approx 01: 48$ UT on May 15, 2013 .

\section{PROPAGATION OF DS IN THE SOLAR WIND}

According to the Wind satellite data, the IMF modulus and the SW density vary in antiphase. The mean correlation coefficient between $N$ and $B$, computed at 01:15-04:00 UT, $r=-0.91$ (Figure 4, $a$ ) allows us to define these structures as DS [Eselevich, Eselevich, 2005]. Having formed in the corona near the solar surface, the DS moves in the solar wind as a unit with retention of spatial irregularities in the SW density (or fractal structure, see below) and the IMF modulus to Earth's orbit. Figure 4, $a-d$ exhibits a sequence of DS observations at different distances from the Sun. The large negative correlation coefficient between the SW density and IMF modulus variations holds to Earth's orbit and farther (Figure 4,c-e), which is evidence of the stability and continued existence of DS macro and microstructures over large distances from the Sun.

A vivid example of the retention of the DS microstructure over large distances from the Sun is given in Figure $4, e, f$. It indicates that the correlation coefficient $r$ even slightly increases as the microDS moves from the Wind orbit to the THB orbit (see $r$ values in bottom panels in Figure 4, $e, f$ ), which implies the stability of the DS microstructure ( $m$ in Figure 5 ) during its propagation.

The DS of interest is located in the ecliptic plane (XY in Figure 1) mainly along the OY axis. Its angle of inclination $\varphi$ to the $\mathrm{OX}$ axis (Figure 1) is determined from the difference between the moments when Wind and ACE began recording characteristic DS features, connected by straight lines in Figure $4, b$. The start of DS recording on ACE is delayed relative to that on Wind by $\Delta t \sim 612 \mathrm{~s}$. At the SW velocity $V \approx 435 \mathrm{~km} / \mathrm{s}$, this corresponds to a displacement $\Delta x=2.66 \cdot 10^{5} \mathrm{~km}$. The distance along the $\mathrm{Y}$ axis between ACE and Wind

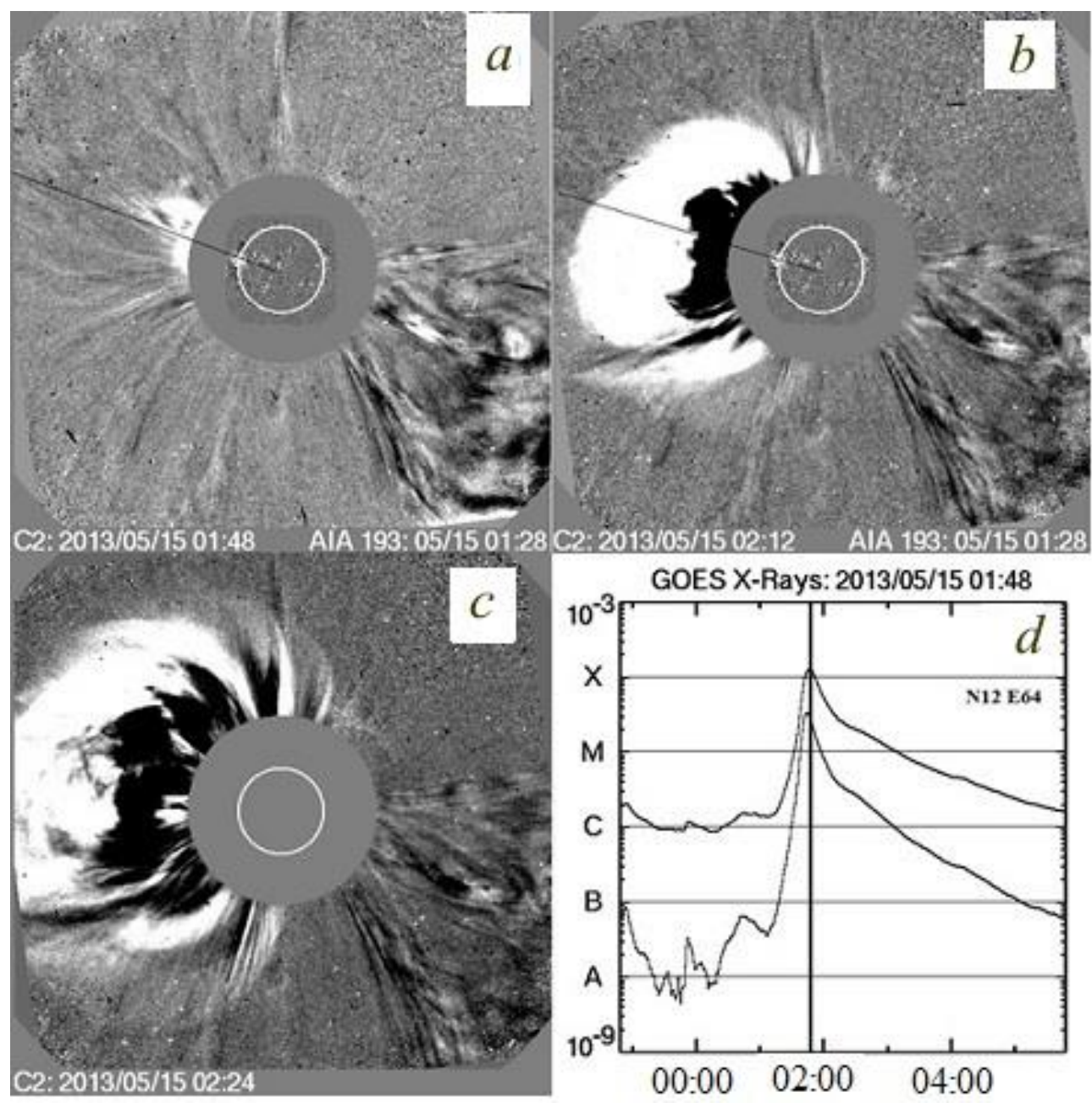

Figure 3. Sequence of difference white-light corona images from the LASCO C2 coronagraph $(a, b, c)$. Inside the white circle corresponding to the solar surface are difference images in the $193 \AA$ channel. X-ray profiles derived from GOES data $(d)$ in ranges 1.0-8.0 $\AA$ (upper) and 0.5-4.0 $\AA$ (lower). The data is from [https://cdaw.gsfc.nasa.gov/CME_list/catalog_description.htm] 

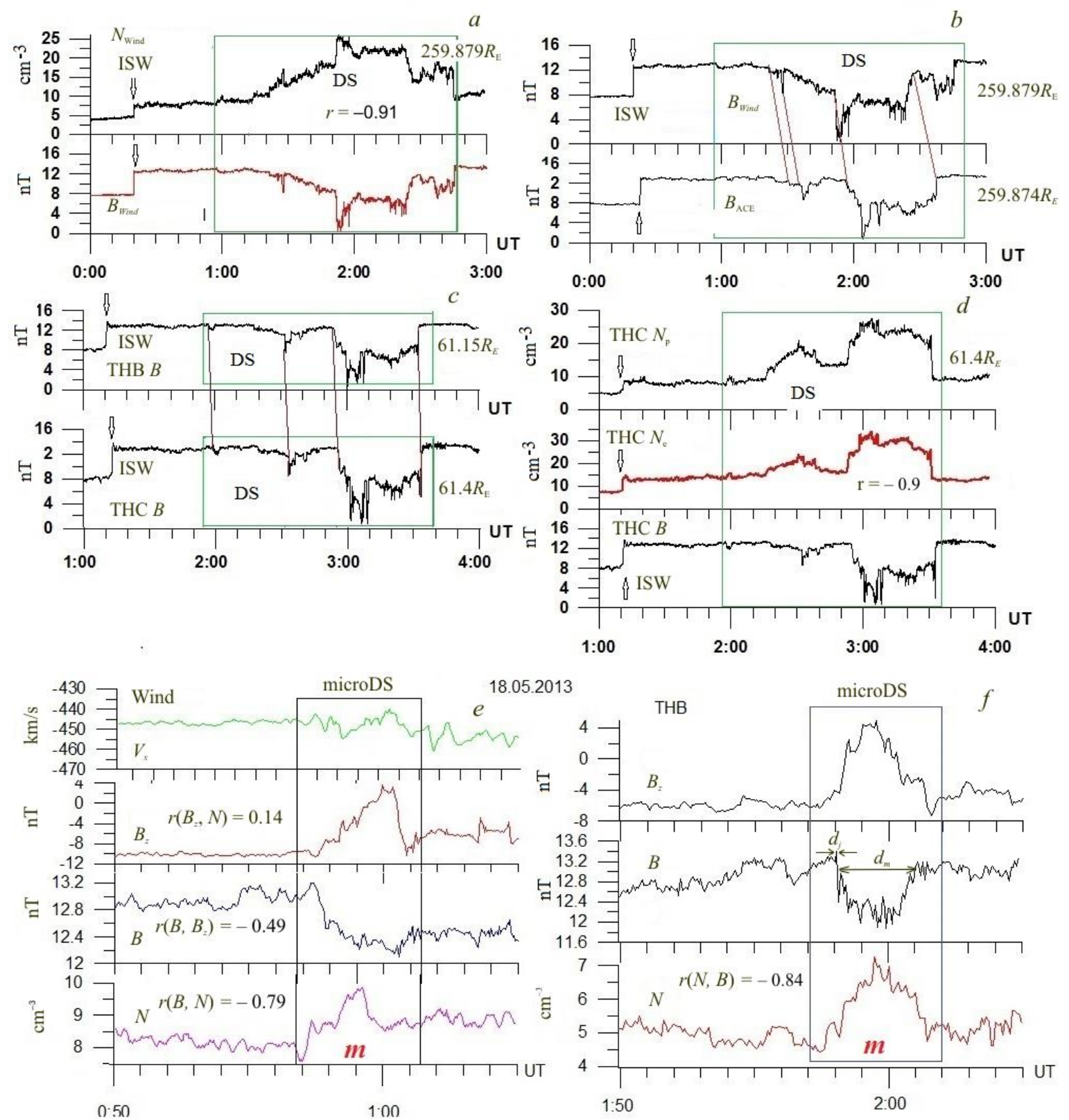

Figure 4. DS propagation in the solar wind: variations in the SW density $N$ and the IMF modulus $B(a)$ according to Wind data; variations the IMF modulus $B$ as derived from Wind and ACE data $(b)$; variations in the IMF modulus $B$ on the THB and THC satellites near Earth's orbit (straight lines connect characteristic details to determine the delay) (c); variations in the IMF modulus $B$, proton and electron concentrations from THC data near Earth's orbit (to the right is the distance in $R_{\mathrm{E}}$ of the corresponding satellite from Earth's center at the time of measurements) $(d)$. The data is from the Website [http://cdaweb.gsfc.nasa.gov/cgi-bin/eval2.cgi]. Retention of the DS microstructure $m$ over large distances from the Sun: SW and IMF parameters in the Wind orbit (e); the same SW region in the THB orbit near Earth $(f)$

$\Delta y=6.97 \cdot 10^{5} \mathrm{~km}$. Hence, $\operatorname{tg} \varphi=2.62$, and the angle of DS inclination to the $\mathrm{X}$ axis $\varphi=69^{\circ}$ (Figure 1). The DS motion with the same velocity in SW near Earth's orbit can be seen from the delay of the same structural details on THB and THC as on Wind and ACE. The distance between the satellites along the $x$ coordinate is $\sim 3 R_{\mathrm{E}}$, which gives a delay of $\sim 1 \mathrm{~min}$ at a speed of $440 \mathrm{~km} / \mathrm{s}$.

\section{RESPONSE IN THE MAGNETOSPHERE}

We examine the dynamics of phenomena in the magnetosphere, using THA, GOES-13, and GOES-15 data on the geomagnetic field and particle fluxes. THA was initially in the dawn sector, moved from the magnetopause toward Earth, and traveled from $9.25 R_{\mathrm{E}}$ to 7.77 $R_{\mathrm{E}}$ during DS observation. GOES-15 was in the afternoon sector (15-20 hr), while GOES-13 was in the premidnight sector (19-24 hr) (Figure 1).

We analyze sequentially the magnetospheric response to the arrival of an interplanetary shock wave (ISW) and DS. Referring to Figure 5, there is a qualitative correlation between the time variation of the plasma density in SW and in the magnetosphere in the time interval corresponding to the arrival of ISW and SW DS in Earth's orbit. After the ISW passage, in the solar wind 
the magnetic field strength $B$ and the ion concentration $N$ increase sharply (Figure 5, curves 1,2). This sharp increase in the parameters results in the storm sudden commencement at 01:10 (SSC, vertical green line in
Figure 5), which is well identified from IL-index variations (Figure 5, curve 9),

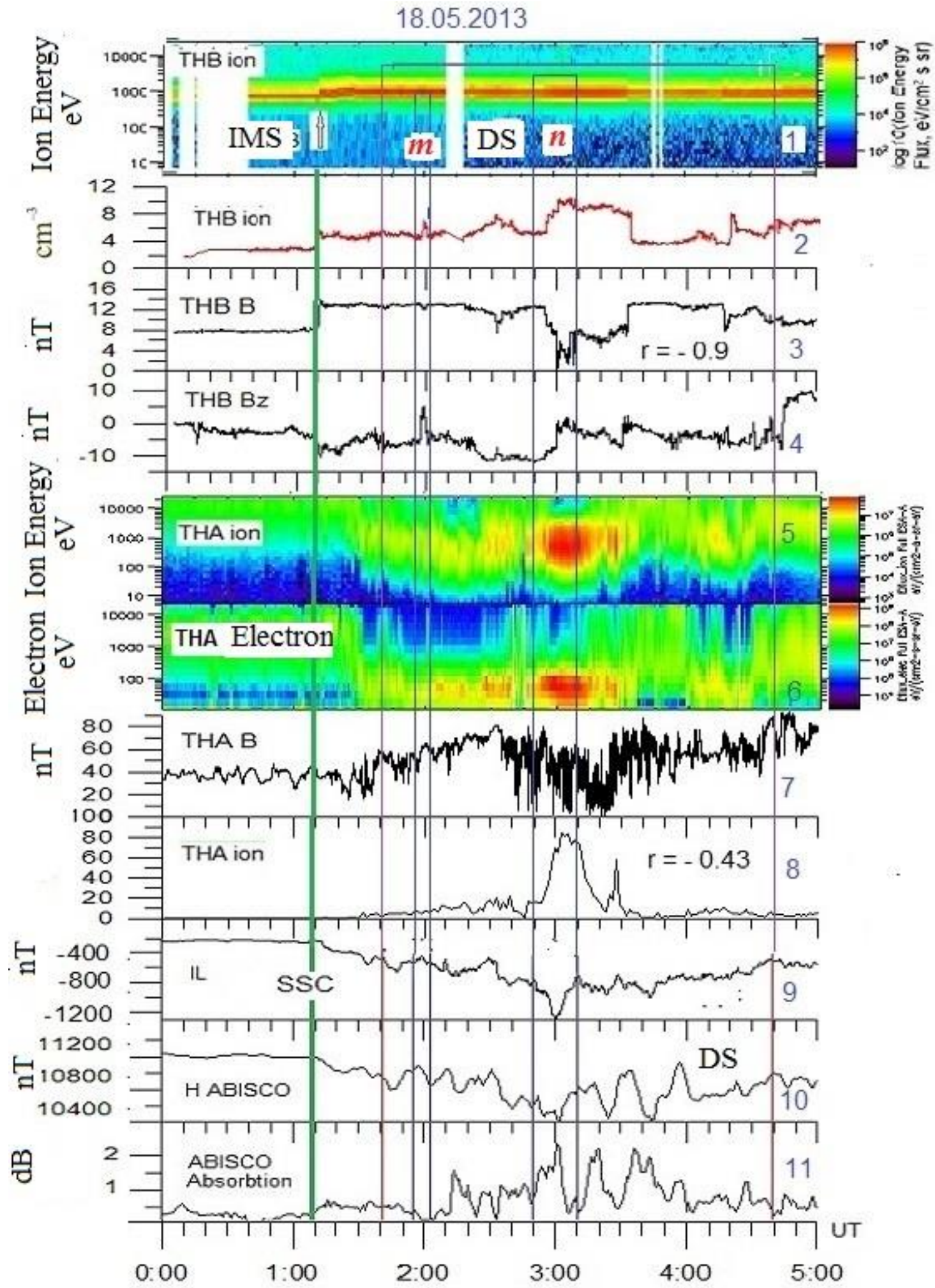

Figure 5. Energy spectrum of ions, variations in ion concentration $N(1,2)$, as well as variations in IMF $B, B_{z}(3,4)$ according to THB data in SW; energy spectra of ions and electrons derived from THA/ESA data $(5,6)$, and variations in the geomagnetic field modulus $B(7)$ and ion concentration $N(8)$ on the THA satellite in the magnetosheath and magnetosphere; $I L$ is the local index of auroral magnetic activity according to the data from the IMAGE magnetic observatory network; variations in the $H$ component of the geomagnetic field (10) and a fragment of a $30 \mathrm{MHz}$ riometer record (11) at the Abisko observatory. The large rectangle marks DS; inside it, the $m$ and $n$ rectangles indicate microDS 
as well as from the magnetogram and riometer records of the Abisko Observatory (Figure 5, curves 10, 11). The jump in magnetic activity can also be seen in $A E$ and $S Y M-H$ variations (Figure $10, a$, curves 8,9 ). After SSC, there is an increase in magnetic activity associated with the magnetosphere compression: $S Y M-H$ increases by $20 \mathrm{nT} ; A E$, by $200 \mathrm{nT}$. The geomagnetic field remains at a constant level without noticeable variations for $\sim 0.5 \mathrm{hr}$. Nonetheless, in the THA orbit in the magnetosphere, no noticeable variations in the plasma density and energy ion and electron spectra (Figure 5, curves 5, 6, 8) are observed after the interaction with ISW.

Before analyzing the magnetospheric response to the arrival of DS, compare characteristic dimensions of DS and microDS $m$ in the radial direction with the size of the magnetosphere $d_{\mathrm{mag}} \approx 50 R_{\mathrm{E}}$ in Figure 1. Figure $2, a$ suggests that the size of large DS $d_{\mathrm{DS}} \approx 180 \mathrm{~s} \times 450 \mathrm{~km} / \mathrm{s} \approx$ $763 R_{\mathrm{E}}$, which is significantly greater than the size of the magnetosphere $d_{\mathrm{mag}}$. The size of microDS $m$ in the time profile (Figure $4, f$ ) is shown by the horizontal line labeled with $d_{m}$ and is as large as $d_{m} \approx 180 \mathrm{~s} \times 450$ $\mathrm{km} / \mathrm{s} \approx 13 R_{\mathrm{E}}$. In this case, the thickness $d_{\mathrm{f}}$ of the leading edge of the microDS $m$, indicated by two horizontal arrows in Figure 4, $f$, (according to higher temporal resolution data): $d_{\mathrm{f}} \approx 7.2 \mathrm{~s} \times 450 \mathrm{~km} / \mathrm{s} \approx 0.5 R_{\mathrm{E}}$. Thus, the microDS size is much smaller than the size of the magnetosphere $d_{\text {mag. }}$. Particularly noteworthy is the small thickness of the leading edge $d_{\mathrm{f}}$, which exhibits a stepwise increase in $N$ and a decrease in $B$. These estimates imply that the DS will most likely flow around the magnetosphere; yet its component microDS $m$ can penetrate through the boundary of the magnetosphere, which may be triggered by a sharp change in the field $B$ at a narrow front $d_{\mathrm{f}} \approx 0.5 R_{\mathrm{E}}$. A similar situation is typical for the $\operatorname{microDS} n$.

In view of the above estimates, we analyze the response of the magnetosphere to the arrival of microDS $n$.

After interaction with the magnetosphere, in microDS $n$ the ion concentration increases to $90 \mathrm{~cm}^{-3}$ in an hour (Figure 5, curve 8), while in the solar wind the maximum ion concentration in microDS $n$ is $\sim 11$ $\mathrm{cm}^{-3}$ (Figure 5, curve 2).

Comparison of data in Figure 5 demonstrating high synchronicity of its changes allows us to conclude that disturbances in the magnetosphere are directly related to the DS impact. Some details of this impact are given in Figures 6 and 7. Figure 6 shows that inside the microDS $n$, recorded near the magnetopause by the THA satellite, there is an electric field pulse $E_{y} \sim 400 \mathrm{mV} / \mathrm{m}$ (Figure $6, b$ ) and equiphase condition for variations of the electric field $E_{z}$ (Figure 6, $a$ ) with a period $T \sim 200 \mathrm{~s}$ and variations in the geomagnetic field modulus $B$ (Figure 6,c) and the proton flux with $E_{\mathrm{p}}=95 \mathrm{keV}$ (Figure $6, a$ ) on the GOES-15 satellite in the geosynchronous orbit. The in-phase variations in the electric field and particle fluxes may indicate a causal link between the phenomena.

An additional confirmation of this is the synchronism of the jump of the electric field components, as well as their subsequent oscillations with the period $T \sim 200 \mathrm{~s}$ on the THA satellite, which coincide with the beginning of microDS interaction with the magnetopause, and oscillations of the geomagnetic field and the energy density of the radiation belt proton flux in the energy range 95-475 $\mathrm{keV}$ in the geosynchronous orbit on GOES-15 (in Figure $6, a E_{\mathrm{p}}=95 \mathrm{keV}$ is marked with the red curve).

Features of this effect can be seen in Figure 7. In the geosynchronous orbit in the afternoon sector (GOES15) in the DS interval, oscillations of the geomagnetic field (Figure 7, $a$, curve 3) and proton fluxes in the energy range 75-575 keV (Figure 7, $a$, curve 4) are recorded. Electron flux fluctuations in the energy range $40-475 \mathrm{keV}$ are insignificant (Figure 7, $a$, curve 2). In the geomagnetic field at 02:48-03:10 UT, a train of oscillations is observed in the Pc5 range of geomagnetic pulsations (Figures $6,7, a$, curve 3 ). In the same time span, there are variations in the electric field components (Figure 6, $a, b$ ) with approximately the same period and proton flux modulation in a $75 \mathrm{keV}$ energy channel (Figure $7, a$, curve 4 ). The strongest proton flux modulation with a period of $\sim 60 \mathrm{~s}$ is in $200-310 \mathrm{keV}$ channels (Figure $7, d$ ). In this energy range, the modulation depth is as great as $90 \%$ (with proton beams observed). However, the greatest proton flux is seen on both satellites in the $140 \mathrm{keV}$ channel.

The initial compression of the magnetosphere caused global SSC (indicated by the arrow) at 01:10 UT (Figure 7, a) [http://www.obsebre.es/en/rapid] and the development of the magnetic storm main phase, which led to a change in radiation belt proton and electron fluxes whose dynamics before the DS manifestation is typical, most pronounced in variations of the electron flux with $E>40 \mathrm{keV}$. Figure 7, $a$ (curve 2) and $d$ indicates that the arrival of the compression wave from the magnetopause generated due to the interaction with ISW causes a sharp increase in electron fluxes, which then, as the storm main phase develops, begin to decrease [Hess, 1972].

Unlike the effect of compression wave propagation in the magnetosphere, the interaction with DS as a sequence of DS microstructures (two of which are denoted by $m$ and $n$ in Figure 7) causes geomagnetic field oscillations in the geostationary orbit (Figure $7, b$, curve 3 ) and an

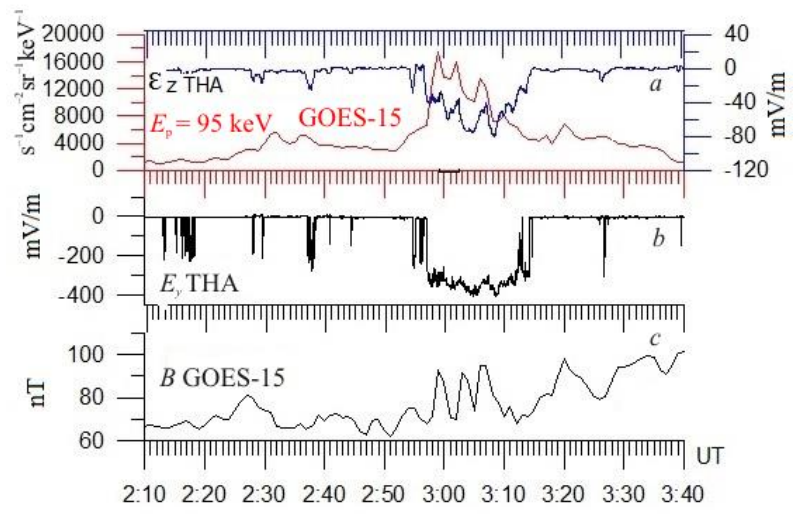

Figure 6. Equiphase condition for variations in the electric field components $E_{z}, E_{y}(a, b)$ near the magnetopause on the THA satellite and variations in the geomagnetic field modulus $B(c)$ and in the proton flux energy density with $E_{\mathrm{p}}=95 \mathrm{keV}$ (a) in the geosynchronous orbit 

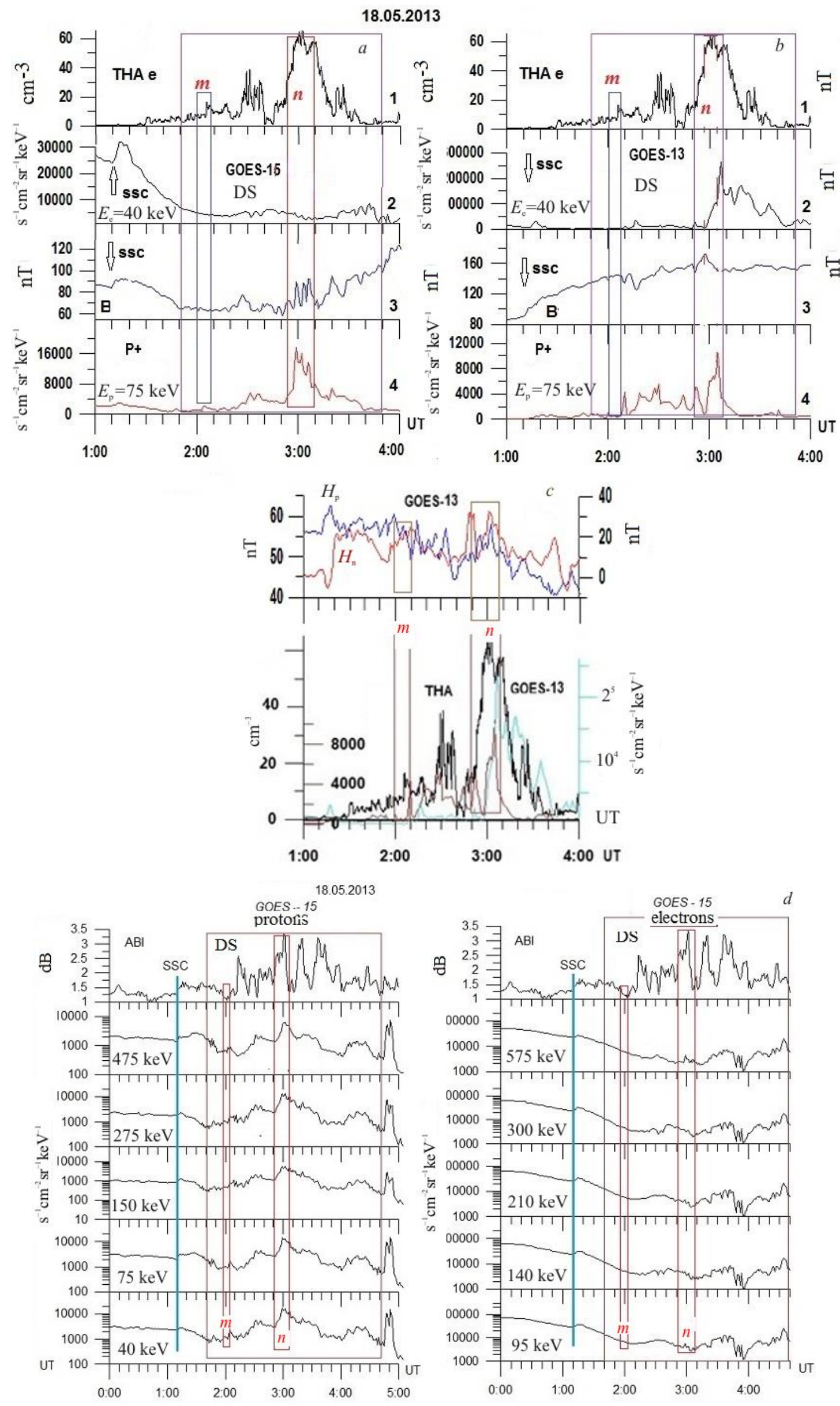

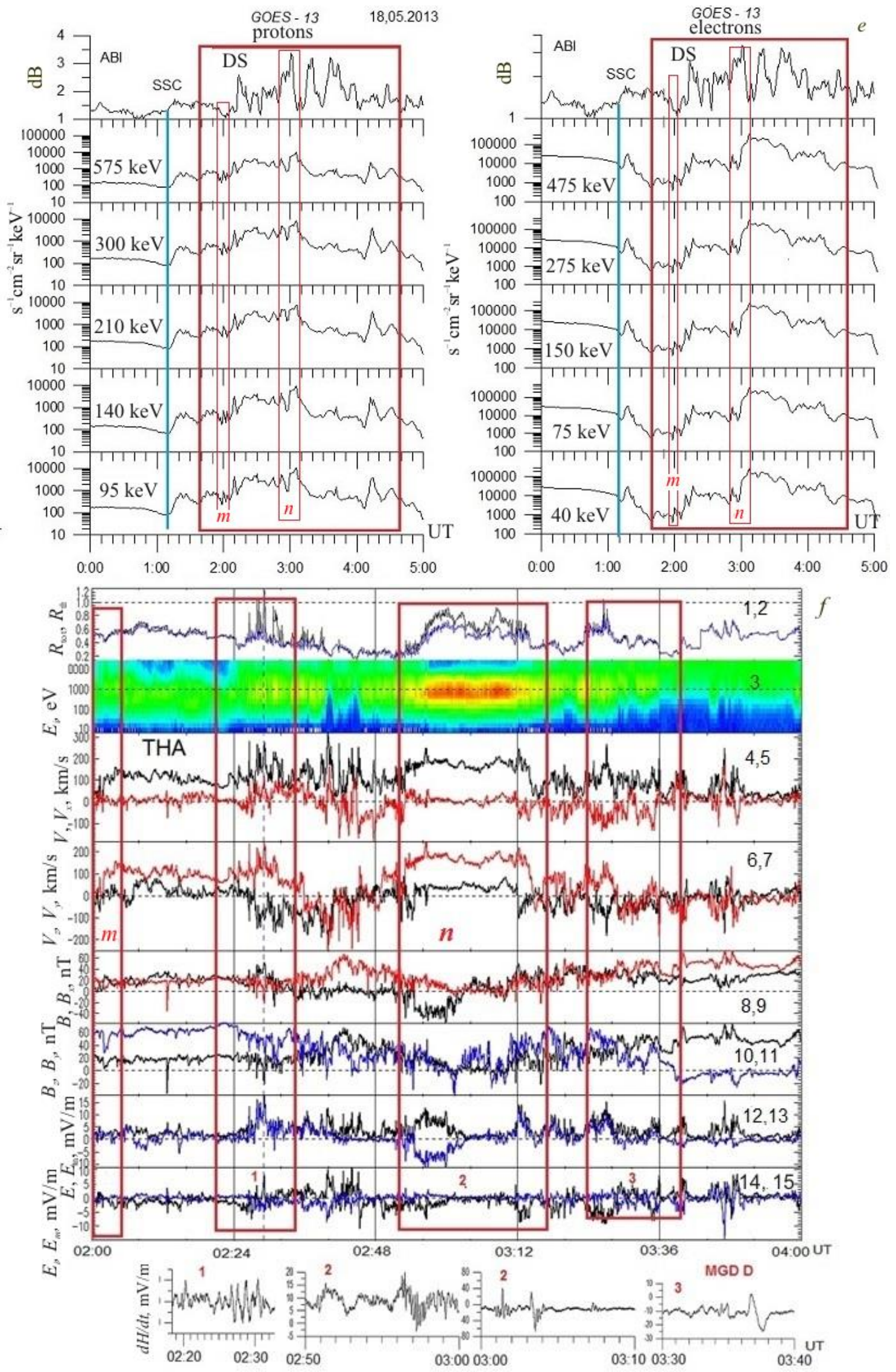

Figure 7. Variations in parameters of the geomagnetic field and the energy density of charged particle fluxes in the magnetosphere, associated with the DS impact on the magnetosphere. The large rectangle marks the DS interval in Earth's orbit corresponding to the DS observation in the solar wind. The letters $m$ and $n$ denote a microDS. Panels $a$ and $b: 1-$ electron density variations on THA; 2, 3, 4 - the energy density of the electron flux with $E=40 \mathrm{keV}$; variations in the modulus $B$ and in the proton 
flux with an energy of $75 \mathrm{keV}$ on GOES-15 (a) and GOES-13 (b) respectively. Panel $c$ illustrates variations in $H_{\mathrm{p}}$ and $H_{\mathrm{n}}$ components of the geomagnetic field on GOES-13 in the magnetotail plasma sheet (top panel) and charged particle fluxes (bottom panel) on the THA (black curve) and GOES-13 (blue and red curves). Panels $d, e$ are variations in the energy density of proton and electron flux in the geostationary orbit during the afternoon (GOES-15) and pre-midnight (GOES-13) hours and in the intensity of auroral absorption at midnight. Panel $f$ displays estimated SW plasma and magnetosheath parameters for DS: 1,2 - ratio of total plasma energy density (black curve) to thermal pressure (blue curve) on THA and in SW on Wind; 3 - energy spectrum of ions on THA; 4, 5 - plasma velocity on THA: black curve - total $V$, red curve $-V_{x \mathrm{GSM}}$ in the geocentric Sun-magnetosphere coordinate system (GSM); $6,7-$ plasma velocity on THA: black curve $-V_{z}$, red curve $-V_{y \mathrm{GSM}} ; 8,9-$ geomagnetic field components on THA: black curve - full $B$, red curve $-B_{x \mathrm{GSM}} ; 10,11-$ geomagnetic field components on THA: black curve $-B_{z}$, blue curve $-B_{y \mathrm{GSM}} ; 12,13$ - electric field on THA: black curve - full $E$, blue curve - earthward $E_{\mathrm{n}} ; 14,15-$ electric field on THA: black and blue curves - magnetopause transverse components $E_{1}$ and $E_{\mathrm{m}}$ respectively; the bottom panel shows fragments of oscillograms from the induction magnetometer of the MGD observatory near local noon

increase in fluxes of electrons and protons trapped in the radiation belt (Figure 7, $b$, curves 2 and 4 ). In this case, duration of these processes coincides with the period of DS observation in the solar wind (large rectangle in Figure 7). It is noteworthy that the dynamics of particle fluxes is synchronous in the solar wind (satellites THB, THC), near the magnetopause (THA) and in the geosynchronous orbit (GOES-13, -15).

The dynamics of phenomena in the geosynchronous orbit in the pre-midnight sector of the magnetosphere differs from the dynamics in the daytime sector primarily in that a sharp increase in fluxes of both protons and electrons occurs on the night side (Figure 7, b, curves 2, 4 and Figure $7, c)$; and only in the proton flux, on the daytime side (Figure 7, $a$, curve 4). It should be emphasized that the period of observations of increased particle fluxes on GOES-13 and -15 coincides with that of increased proton and electron concentration on THA in the magnetosheath near the magnetopause. On GOES13 , the maximum proton and electron fluxes lag behind the maximum ion concentration on THA (Figure 7, $c$, bottom) and the maximum proton flux on GOES-15 (Figure 7,d).

Another important detail is seen in Figure 7, $c$ in comparison of $B_{x}$ and $B_{z}$ variations with the electron flux burst on GOES-13, which crossed the plasma sheet. The components change in phase, which indicates the absence of geomagnetic field dipolization, which would lead to an increase in the particle flux from the magnetotail.

There is also indirect evidence of the passage of plasma from the magnetosheath to the magnetosphere, associated with the generation of plasma jets in the magnetosheath [Dmitriev, Suvorova, 2015]. In that article, an empirical threshold value of plasma velocity $V=220 \mathrm{~km} / \mathrm{s}$ in these jets has been obtained. For the DS-magnetosphere interaction of interest, plasma parameters in the magnetosheath from 02:00 to 04:00 UT have been calculated. The calculation results presented in Figure $7, f$ suggest that at $\sim 2: 25$, at 02:47-03:13, and at $~ 03: 35$ UT THA observes sharp increases in the energy density $R_{\text {tot, }}$ related to plasma jets whose speeds are above the threshold, i.e. the necessary condition for penetration of the magnetosheath plasma into the magnetosphere is fulfilled. However, we have no direct data on this since there was no THEMIS satellite in the magnetosphere that could register the penetration of plasma.

The plasma jets with earthward high velocities are observed in the region of the microDS $n$ boundaries. The duration of the peaks is $\sim 1 \mathrm{~min}$, which at a velocity of $\sim 200 \mathrm{~km} / \mathrm{s}$ yields a characteristic size of $\sim 2 R_{\mathrm{E}}$, which is much smaller than the transverse size of the magnetosphere $\sim 30 R_{\mathrm{E}}$. Thus, these small-scale structures can "pierce" through the magnetopause, ensuring penetration of magnetosheath plasma into the dayside magnetosphere. The interaction of jets with the magnetopause is also a source of Pc4-5-Pi2 pulsation bursts, which we observe on the day side at the MND, MGD, PET observatories (Figures $7, f, 8, b$ ). Thus, we can see that the plasma jets of the magnetosheath do interact with the magnetopause, and this interaction is most likely to occur with penetration of the magnetosheath plasma into the dayside outer magnetosphere.

When DS penetrates into the magnetosphere, its velocity decreases from $V=450 \mathrm{~km} / \mathrm{s}$ in the solar wind to $V=175 \mathrm{~km} / \mathrm{s}$ in the orbit segment of THA-GOES$15 R_{1}=(1 \div 0) R_{\mathrm{E}}$ and to $V=72 \mathrm{~km} / \mathrm{s}$ in GOES-15-GOES$13 R_{2}=(0 \div-6.5) R_{\mathrm{E}}$. At the same time, the range of proton concentration variation increases. This result is in line with the conclusions drawn in [Dmitriev, Suvorova, 2015; Rakhmanova et al., 2015] about an increase in the amplitude of the density front in the transition layer 1.5 times and a decrease in the jet velocity to $270 \mathrm{~km} / \mathrm{s}$ compared to the velocity in SW.

The calculation results presented in Figure $7 f$ show that at $\sim 2: 25,02: 47-03: 13$, and 03:35 UT THA observes sharp increases in the energy density $R_{\mathrm{tot}}$, associated with jets. These increases are determined by the parameter $\beta_{\mathrm{k}}>1\left(\beta_{\mathrm{k}}=1 / 2 \rho u^{2} / B^{2} / 2 \mu_{0}\right.$, where $\rho$ is the plasmoid density; $B$ is the strength of the surrounding magnetic field; $\mu_{0}$ is the magnetic constant; $u$ is the jet velocity [Dmitriev, Suvorova, 2015]. The jets that interacted with the magnetopause are a source of the Pc4-5$\mathrm{Pi} 2$ pulsation bursts, recorded on the day side at the MND, MGD, PET observatories. As a result of the interaction of jets with the magnetopause, the magnetosheath plasma could penetrate into the dayside outer magnetosphere. Fragments of Pi2 oscillograms from the induction magnetometer of the MGD observatory $(\mathrm{MLT}=\mathrm{UT}+10)$ near the local noon are given at the bottom of Figure 7, $f$.

\section{FEATURES OF THE GEOMAGNETIC RESPONSE}

Features of the geomagnetic response to the interaction of SW DS, shown in Figure 2, with the magnetosphere are displayed in Figures 8-11. According to OMNI data [http://cdaweb.gsfc.nasa.gov/cgibin/eval2.cgi], during the four-hour period preceding the 
ISW arrival there was low planetary $\left(K_{\mathrm{p}}=2\right)$ and moderate auroral $\left(A E_{\mathrm{av}}=385 \mathrm{nT}\right)$ magnetic activity corresponding to the conditions in the slow solar wind: $V_{\mathrm{av}}=373$ $\mathrm{km} / \mathrm{s}, N_{\mathrm{av}}=4.4 \mathrm{~cm}^{-3}$, the IMF vertical component was negative $B_{z \mathrm{GSMav}}=-2.96 \mathrm{nT}$ (Figure 3 ). These conditions, according to today's generally accepted models [Akasofu, 2017], determine the energy pumping into the magnetotail due to reconnection of the interplanetary and geomagnetic fields.

A jump in the SW and IMF parameters at 01:10 UT at the interplanetary shock front provokes a sudden commencement of a moderate magnetic storm with maximum $D s t=-61 \mathrm{nT}$ with a very short $(\sim 10$ min) phase of disturbance by a corpuscular flux (Figure $10, a$, curves 5,6 ).

During the growth phase of the event under study, a similarity is observed in the response to the magnetosphere-ISW interaction in the geostationary orbit and on
Earth as a sharp jump in the total magnetic field on GOES-15 and a positive global sharp increase in the horizontal magnetic field component $H$ on Earth's surface (vertical line in Figure 8, $a$ ).

Responses to propagation of the magnetized DS plasma into the magnetosphere differ in the dayside, dusk, and nightside sectors of the magnetosphere; therefore, we will analyze them one by one.

\section{DAYSIDE SECTOR}

In the dayside magnetosphere (Figure 8, $a$, curves 5-7), variations in the $H$ component of the geomagnetic field at observatories from the equator to the auroral zone are determined by the variation in the DS magnetic field modulus (Figure 8, a, curve 2). Correlation coefficients between $H$-component variations for pairs of observatories
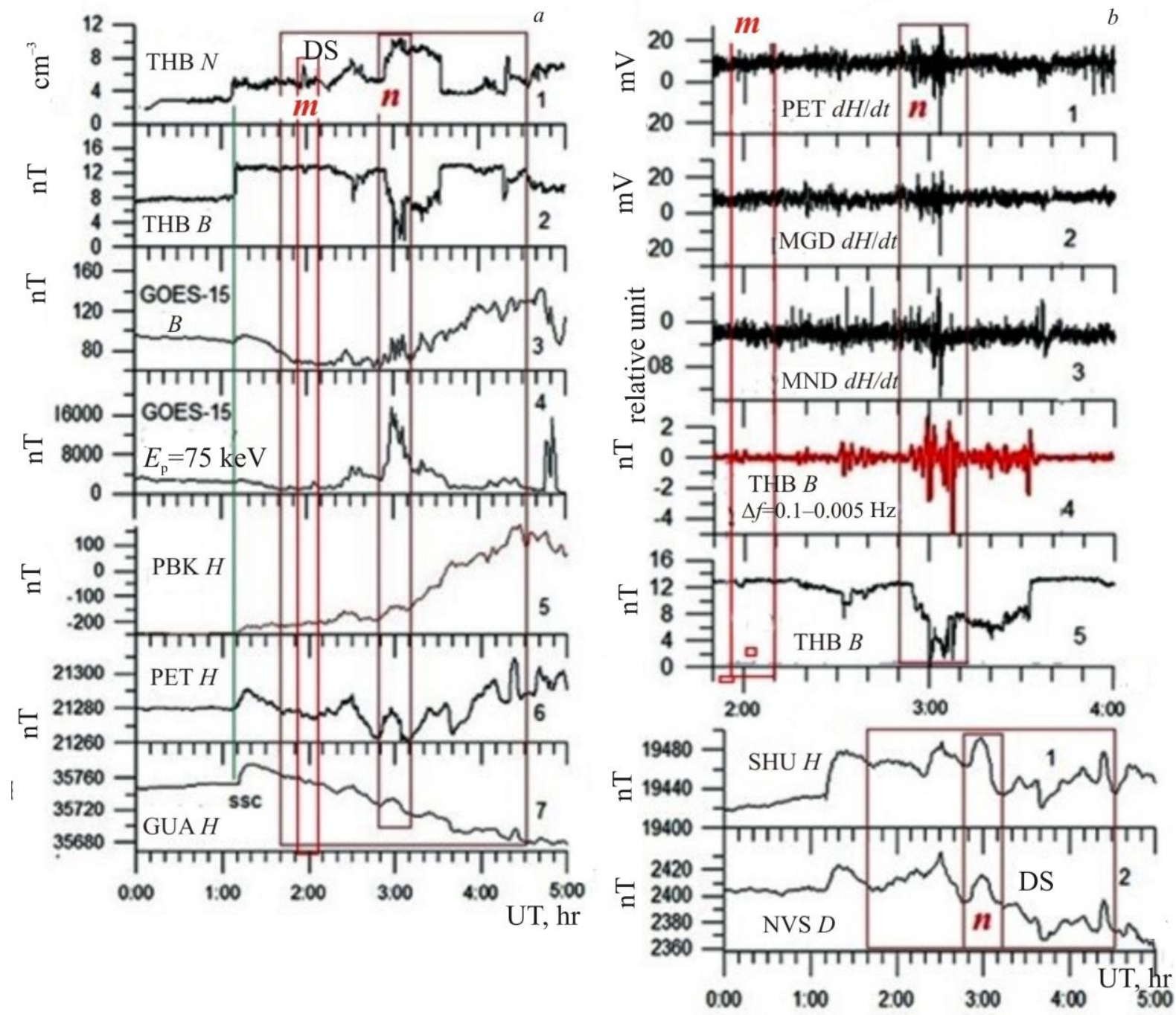

Figure 8. Panel $a$ depicts variations in the SW plasma density on THB (1) and in the IMF modulus $B$ on THB (2) and GOES15 (3), as well as in the proton flux on GOES-15 (4); fragments of $H$-component magnetograms from auroral (5, PBK), midlatitude (6, PET), and low-latitude (7, GUA) observatories at near-noon local time. The green vertical line indicates SSC; the large red rectangle corresponds to the interval of DS observation in the solar wind. Panel $b$ shows fragments of oscillograms from induction magnetometers (01:50-04:00 UT) of the mid-latitude observatories PET, MGD, MND, spaced in longitude by $54^{\circ}(1-$ 3 ), and IMF modulus $B$ variations on THB $(4,5)$. Panel $c$ presents fragments of magnetograms from the ground-based observatories SHU ( $H$ component) and NVS ( $D$ component), located at latitudes of $54^{\circ}$ and $55^{\circ}$ and spaced in longitude by $150^{\circ}$ 
GUA-PET and PET-PBK $r=0.62$ and $r=0.91$ respectively. The $H$-component variations according to ground-based measurements correlate well with magnetic field modulus $B$ variations in the geostationary orbit and in the solar wind. All these magnetic observatories record a response to the interaction between the magnetosphere and CME, which includes ISW, sheath, and a diamagnetic structure consisting of five successive microstructures. The magnetic storm began (SSC) with a sharp increase in the $H$ component (at GUA, by $30 \mathrm{nT}$; at PET, by $13 \mathrm{nT}$; and at PBK, by $34 \mathrm{nT}$ ), caused by the magnetosphere-ISW interaction. The next increase in the $H$ component at the ground-based observatories (at GUA, by $9 \mathrm{nT}$; at PET, by $16 \mathrm{nT}$; at PBK, by $24 \mathrm{nT}$ ), comparable in magnitude with its jump during SSC (Figure 8, $a$, curves 5-7), and subsequent geomagnetic field variations are linked to its interaction with the microDS $m$ at 01:58 UT and $n$ at 02:50 UT.

The effect of the microDS-magnetosphere interaction is most pronounced in the interval marked with the rectangle labelled $n$ in Figure 8, $a$. The beginning of the microDS $n$ passage at 02:55 UT, determined from the antiphase variation in $B$ and $N$ on THB (curves 1,2 in Figure 8 ), is reflected in the excitation of Pc5 oscillations of the geomagnetic field modulus in the geostationary orbit on GOES-15 (curve 3), a sharp fluctuating increase in the proton flux in the energy range 75-475 $\mathrm{keV}$ (curve 4), and a sharp synchronous increase in the geomagnetic field components overlapping with bursts of Pc4-5-Pi2 geomagnetic pulsations at observatories of the illuminated hemisphere from the equator to auroral latitudes (GUA-PBK, curves 5-7) in the longitudinal range 07-19 MLT $\left(83^{\circ}-199^{\circ}\right.$, NVS-SHU, Figure 8, $c$ ).

The DS-magnetosphere interaction manifested itself not only in the amplification of ionospheric currents generating similar geomagnetic field variations from the equator to the auroral zone, but also in the passage of MHD waves from SW observed without delay in widelyspaced mid-latitude observatories $\left(\Delta \Lambda=54^{\circ}\right)$ in the form of Pc4-5-Pi2 (100-200 s) geomagnetic pulsations (Figure $8, b$, curves $1-4)$. Synchronism of the excitation of geomagnetic pulsations, as well as synchronism of variations in ionospheric currents, which induce geomagnetic field variations, in a large latitude-longitude range, indicate the remoteness of the source.

Arguments for the assumption about wave propagation into the magnetosphere from SW to Earth may be both the similarity between dynamic spectra of bursts of the pulsations and the same frequency $(\sim 0.0055 \mathrm{~Hz})$ of maxima (indicated by arrows) of the spectral density of magnetic field oscillations at ground-based observatories and on the THB and THC satellites in SW (Figure 9).

Relying on the similarity between oscillograms of oscillations and dynamic spectra of pulsation bursts in SW and at ground-based observatories in the dayside magnetosphere in the $104^{\circ}-199^{\circ}$ longitudinal range, we can conclude that there are geomagnetic field variations generated by the same distant source. This source consists of fluctuations of the magnetic field modulus and plasma density in DS (fractal components of DS), driven by CME near the Sun and transferred by SW to
Earth's orbit, while retaining their structure. The DS crossed the bow shock and, perhaps, the magnetopause, and its fine structure was observed on the day side from dawn to dusk hours as Pc4-5-Pi2 geomagnetic pulsations.

The passage of waves of this frequency range from SW into the magnetosphere has been reported in [Mishin, 1996; Kessell et al., 2004; Potapov, Polyushkina, 2010; Klibanova et al., 2016].

In the dayside magnetosphere, the DS microstructure effect also appears as fluctuations of the proton flux in the energy range $95-475 \mathrm{keV}$ in the radiation belt, which are synchronous with the magnetic field oscillations on GOES-15 (Figure. 8, $a$, curve 4) and are in antiphase with the magnetic field modulus oscillations in the solar wind DS (Figure 8, $a$, curves 1,3 ). There are no noticeable variations in the electron flux in the range 40-575 keV (Figure 7, a, curves 2, 3, 4).

\section{DUSK-MIDNIGHT SECTOR}

In the nightside magnetosphere, the interaction with DS caused amplification of ionospheric currents (current system DP2). In the dusk-midnight sector, both the westward and eastward electric jets sharply intensify (ellipse and arrows above the PINA and FCHU observatories in Figure 10,a) [Gjerloev, 2012].

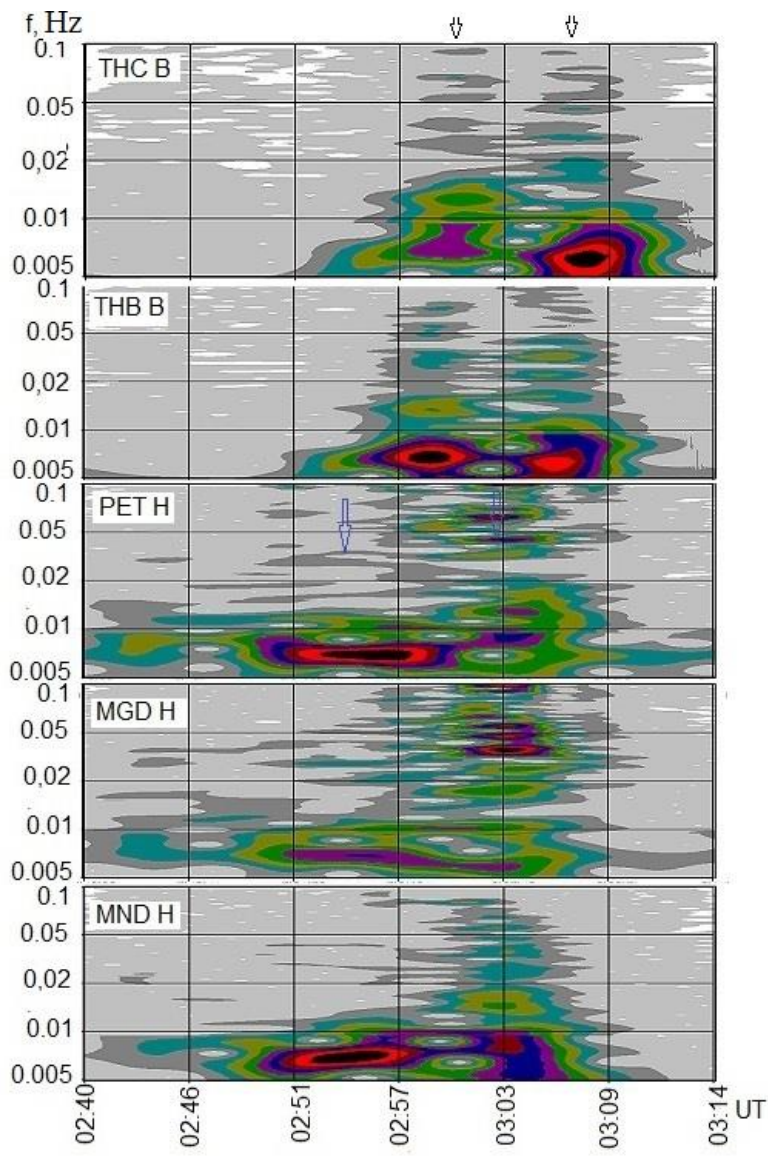

Figure 9. Dynamic spectra of IMF $B$ oscillations on THC and THB and of the geomagnetic field horizontal component $H$ at the mid-latitude observatories PET, MGD, MND, located in the near-noon sector (10-02 MLT), whose oscillograms are presented in Figure 8, $b$. The onset of geomagnetic pulsations on Earth is ahead of the onset of pulsations on THB and THC 
Features of the magnetospheric disturbance in the duskmidnight sector (19-23 MLT) are shown in Figure 11. It can be seen that at the PINA observatory, located under the ground projection of the eastward current, when interacting with DS (microDS $m$ in Figure 5) pulsations with a nonstationary spectrum (irregular pulsations of diminishing periods, IPDP) are generated (Figure 11, $d$ ). The mechanism of excitation of such pulsations is the cyclotron instability of protons linked to the injection of protons with $E \sim 30-50 \mathrm{keV}$ into closed magnetic shells [Guglielmi, 1979]. In the work cited, it is assumed that protons are injected from the plasma sheet of the magnetotail at the breakup of the substorm expansion phase. In the case of interest, however, the FCHU and PINA magnetograms (Figure 11,e) exhibit no sharp negative change in the $H$ component, which indicates the breakup of the expansion phase.

Moreover, the onset of IPDP generation coincides with the onset of an increase in the proton flux in the energy range $E=75-475 \mathrm{keV}$ in the geostationary orbit (see Figure $7, a, d$ ), which, in turn, is connected with the recording of increased fluxes of magnetosheath plasma on the THA satellite. At the same time, at the FCHU observatory, located on the same meridian with PINA but $8.5^{\circ}$ to the north, under the westward jet simultaneously with the onset of IPDP, powerful bursts of Pi1-2 pulsations are recorded, which are a generally recognized indicator of substorm onset. Generation of such pulsation bursts is associated with precipitation of $>10 \mathrm{keV}$ electrons [Mishin et al., 2020]. Pay attention to an important point. The onset of the above phenomena coincides with the passage of the DS microstructure $m$ into the magnetosphere. The moment of its interaction with the magnetosphere is marked with the rectangle $m$ in Figure 5 and with the arrow in Figure 11, $b, d$. It is noteworthy that, as seen in Figures 4, $e$ and 5, the DS microstructure $m$ is accompanied by an abrupt short-term (four minute) change of the direction of the IMF $B_{\mathrm{z}}$ from south to north (from -6 to +4 nT). In [Kokubun et al., 1977; Hsu and McPherron, 2002], a significant relationship has been found between the northward turn of $B_{\mathrm{z}}$ and the breakup of the substorm expansion phase. This northward turn of the vertical component could be considered the onset of the substorm if there were all its components; yet one of the main elements of the substorm is absent - the amplification of the DP-2 current system. Moreover, the $A E$ index, which is the main indicator of the substorm current system intensity, decreased from $646 \mathrm{nT}$ at 02:00 UT to $527 \mathrm{nT}$ at 02:10 UT (Figure 10, $b$, curve 9).
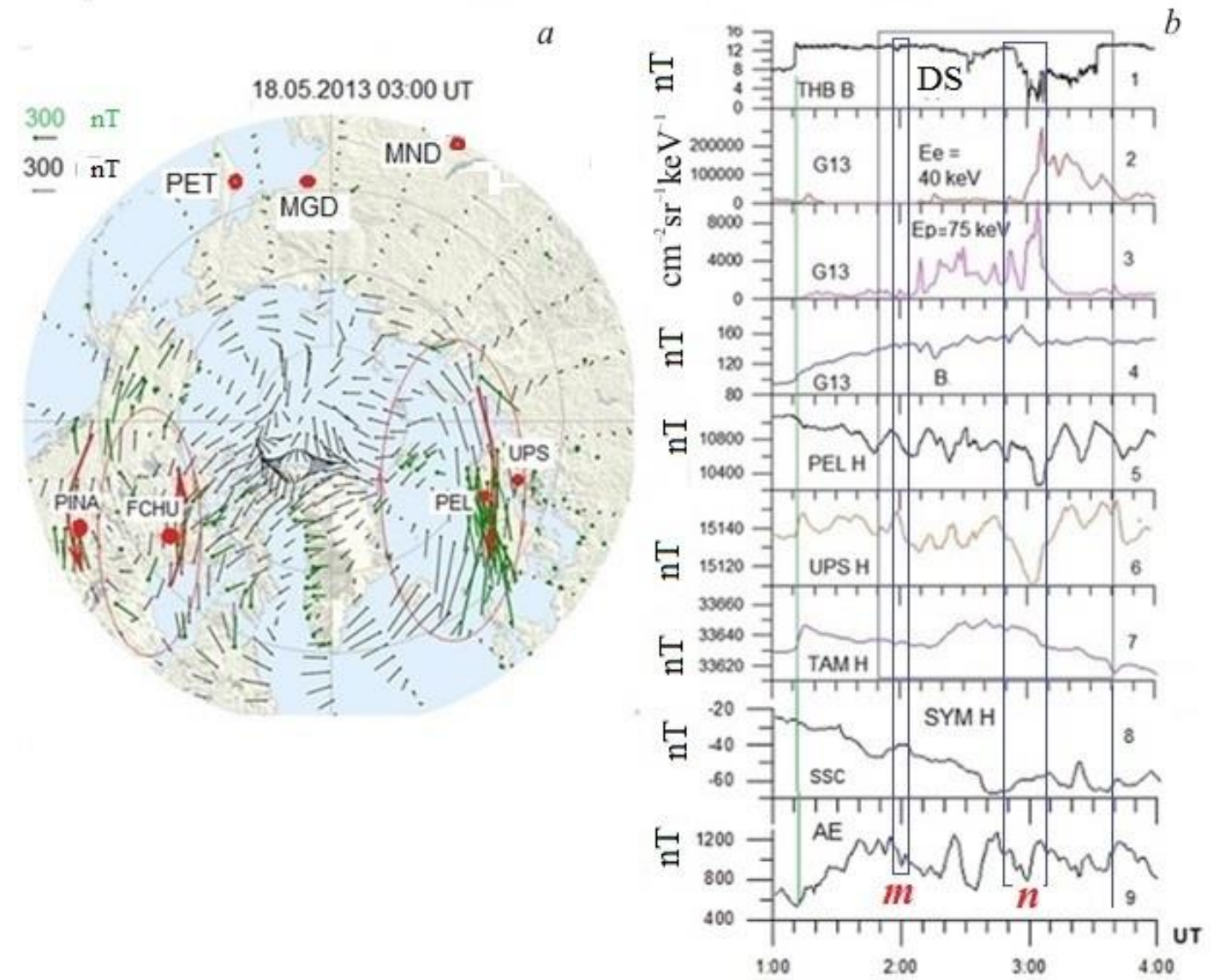

Figure 10. Panel $a$ presents a vector diagram of equivalent ionospheric currents according to data [http://supermag.jhuapl.edu/mag]. Locations of the stations are marked with circles. Panel $b$ depicts variations of the magnetic field modulus $B$ in the solar wind (THB, curve 1) and in the geostationary orbit (GOES-13, curve 4), of the electron and proton flux energy density in the geostationary orbit (GOES-13, curves 2, 3), and of the $S Y M-H(8)$ and $A E$ (9) indices, as well as fragments of $H$-component magnetograms from auroral (PEL, 5), mid-latitude (UPS, 6), and low-latitude (TAM, 7) observatories in the post-midnight sector 04-06 MLT. The vertical line indicates SSC; the large rectangle corresponds to DS in SW; rectangles labelled $m$ and $n$, to microDS 


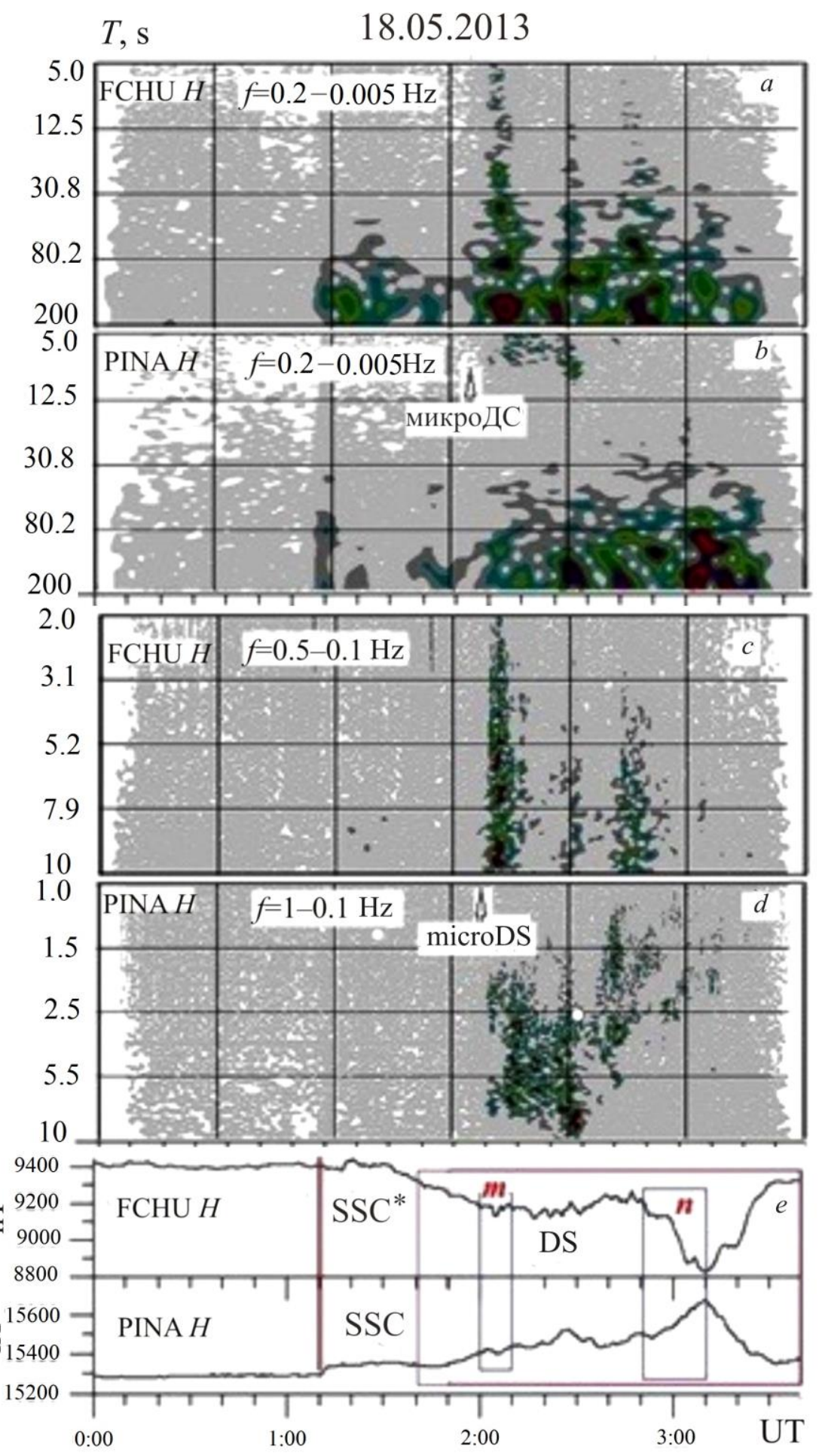

Figure 11. Spectrograms of geomagnetic pulsations in two frequency ranges at the FCHU observatory, located under the westward current $(a, c)$, a negative geomagnetic bay of $\sim 400 \mathrm{nT}$ beginning at $\approx 02: 50 \mathrm{UT}$, and at the PINA observatory, located under the eastward current $(b, d)$, a positive geomagnetic bay of $\sim 300 \mathrm{nT}$, and fragments of the $H$-component recordings from these observatories $(e)$. The letters $m$ and $n$ denote a microDS bay of $\sim 300 \mathrm{nT}$, and fragments of the $H$-component recordings from these observatories $(c)$. The letters $m$ and $n$ stand for a microDS

The data presented in Figures 5 and 11, a-e can be interpreted as follows. The microDS $m$, which causes the northward turn of $B_{\mathrm{z}}$, becomes a trigger for substorm-like phenomena - bursts of Pi1-2 geomagnetic pulsations and IPDP. A source of these pulsations may be electrons and protons injected from the magnetotail plasma sheet, which is confirmed by the enhancement of particle fluxes in it (Figures 1, 7,c), observed at that moment by GOES-13. In addition, for this time interval the CHBG keogram shows auroral activation on the northern horizon or activation in a compressed oval (Figure 12). On the whole, this activation of auroral processes caused by the microDS $m$-magnetosphere interaction may be called pseudo-breakup [Koskinen et al., 1993, Yahnin et al., 2001]. 


\section{AFTERNOON-DAWN SECTOR}

The vector diagram of ionospheric currents (Figure $10, a)$ shows that in the post-midnight-dawn sector $(01-$ 08 MLT), the westward current, which induces a magnetic bay with a maximum of $500 \mathrm{nT}$ at 03:10 UT, sharply increases (Figure 10,b). At the same time, the maximum auroral absorption is recorded at the $\mathrm{ABI}$ observatory (Figure 5, curve 11). Modulation of currents and auroral absorption with close periods with a correlation coefficient $r(A, H)=-0.68$ between them is also observed, which may lead to the conclusion about precipitating electron modulation of the magnetospheric substorm ionospheric current [Belakhovsky et al., 2019]. Indeed, GOES-13 recorded modulated bursts of electron fluxes in the energy range $40-475 \mathrm{keV}$ (Figure $7, b, c, e)$. These observations allow us to classify the substorm as sawtooth [Troshichev et al., 2011].

Temporal dynamics of the geomagnetic field variations and the auroral absorption is in antiphase, and the maximum value of the variation and, accordingly, of the $A E$ index coincides with the smallest value of the modulus $B$ on the THB satellite (Figure 5, curves 5, 10, 11; Figure 10, $b$, curves $1,5,6,9$ ).

The difference of the geomagnetic disturbance considered from the classical substorm is confirmed by the analysis of auroral observations near local midnight (MLT=UT-5), presented in the keogram from the CHBG observatory (Figure 12). While the all-sky camera was turned on after the beginning of the microDS $m$-magnetosphere interaction, auroral activation in the compressed oval can be seen at 02:05-02:25 UT. The airglow activation coincides in time with bursts of geomagnetic pulsations and a short-term ( $\sim 3 \mathrm{~min})$ amplification of the ionospheric current (by $\sim 60 \mathrm{nT}$ ) at the PINA and FCHU observatories (Figure 10, $a$ ), located to the west of CHBG by $20^{\circ}$. The aurora polaris pattern shows evidence for a double oval. The interval
02:50-03:10 UT can be interpreted as the substorm onset, assuming that its auroral breakup was to the east of CHBG. However, at the magnetic observatories located to the east of the auroral observation point, we could not detect bursts of Pi2 geomagnetic pulsations, which are the generally accepted indicator of the breakup and the substorm onset. In the keogram, the double oval shifts to low latitudes from 03:00 to 03:30 UT, as during the development of the substorm. In the course of this shift, the double oval disappears, i.e. the auroras at the equatorial and polar boundaries merge. These observations allow us to state that substorm activity began at 02:50 UT. Duration of the substorm-like phenomena coincides with the duration of DS in the solar wind and magnetosheath/magnetosphere ( 170 min), and the maximum intensity of all components is recorded at 02:50-03:10 UT (designated as $n$ ). Nonetheless, it is caused by the DS-magnetosphere interaction, rather than by the phenomena in the magnetotail. The auroral dynamics confirms the validity of the classification of the substorm disturbance as a sawtooth substorm since magnetic sawtooth disturbances begin against the background of high auroral activity and the double auroral oval structure is typical for them [Troshichev et al., 2011].

In the low-latitude observatory TAM, as well as on the day side, there is a sharp increase in the $H$ component by $14 \mathrm{nT}$, comparable in magnitude with the jump in the $H$ component by $16 \mathrm{nT}$, caused by the interaction with ISW (Figure 10, $b$, curve 7). As the jump in the $H$ component during SSC is the result of the ISWmagnetosphere interaction and is caused by the magnetosphere compression and the current amplification at the magnetopause, we can hold that the sharp increase in the $H$ component of the geomagnetic field, driven by the interaction with DS, also results from the current amplification at the magnetopause due to the plasma

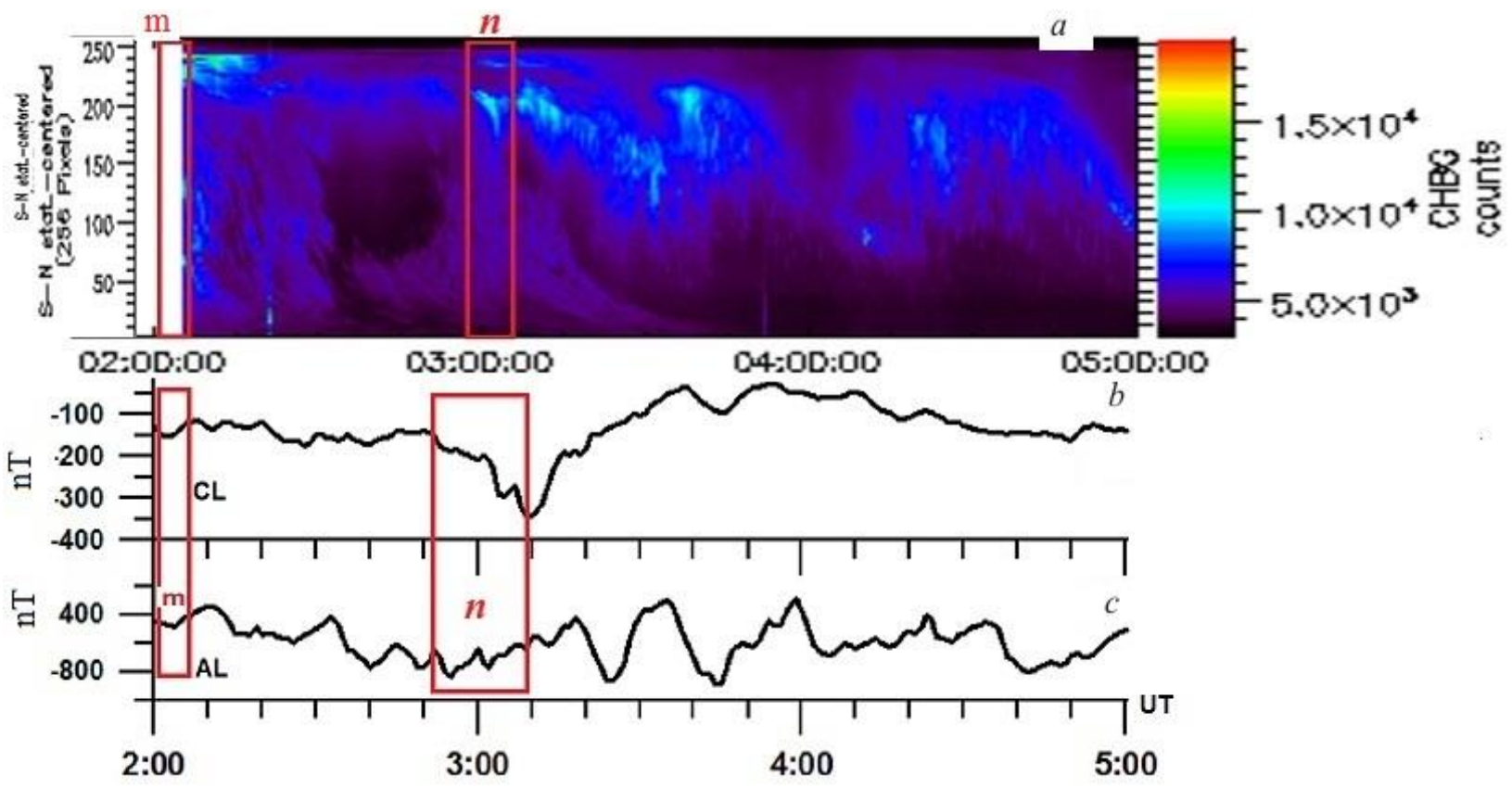

Figure 12. Keogram of auroras from the CHBG observatory (a), as well as variations in the $C L(b)$ and $A L(c)$ indices. The letters $m$ and $n$ stand for a microDS 
density increase in DS. This fact has been emphasized in our paper [Parkhomov et al., 2018].

According to [Shadrina, Starodubtsev, 2016], the passage of an interplanetary shock wave almost always causes substorm disturbances. However, Liou et al. [2003], using large statistics, have shown that ISW in $52 \%$ of cases induce negative magnetic bays (called "compression bays"), but not auroral breakups. In the event considered, no pronounced negative magnetic bay is observed in the magnetograms from the INTERMAGNET observatories in the dusk and night sectors. The $A L$-index variations exhibit no decrease, and $A E$ increases abruptly from 400 to $860 \mathrm{nT}$ and remains at an average level of $660 \mathrm{nT}$ until the DS begins to interact with the magnetosphere at 01:40 UT [http://cdaweb.gsfc.nasa.gov/cgibin/eval2.cgi].

According to [http://carisma.ca/carisma-data/fgmpi2-dbase], at PINA (MLT=UT-6.35), Pi2 pulsations, which are indicators of the breakup and substorm onsets, were recorded at 02:21:44 and 02:50:32 UT. The former moment corresponds to the beginning of the DSmagnetosphere interaction; and the latter, to the beginning of the microDS $n$-magnetosphere interaction, as well as to the onsets of the negative bay recorded at FCHU (Figure 11,e) and the substorm in auroras (Figure 12). Note that $\mathrm{Pi} 2$ pulsations, which are well identified in FCHU data, are not detected by PINA, which is another confirmation of the unusual nature of this substorm activation. Thus, the global dynamics of the DSmagnetosphere interaction in the event considered demonstrates the simultaneous development of a compression pseudobreakup on the dusk side (18-23 MLT) and a sawtooth substorm without signs of breakup in the nightside magnetosphere with a maximum intensity in the 01-05 MLT sector (Figures 11, 12). The onset of the pseudobreakup at $\sim 2$ UT is caused by the microDS $m$ magnetosphere interaction, accompanied by a shortterm northward turn of IMF $B_{z}$, which is a trigger of the short-term plasma flux from the magnetotail. The onset of the sawtooth substorm at $\sim 02: 58 \mathrm{UT}$, its development and duration are determined by the DS energy input from the dayside magnetosphere.

\section{OBSERVATIONAL RESULTS AND DISCUSSION}

Studies of the motion of the SW diamagnetic structure as part of CME have identified features of DS propagation in the solar wind and the effects of its interaction with the magnetosphere, observed in near-Earth space and on Earth's surface.

1. The diamagnetic structure, determined from the high negative correlation coefficient between the IMF modulus and the SW particle concentration, having originated on the Sun during a solar flare is transferred by SW to Earth's orbit with retention of its fine structure. It is a sequence of smaller-scale microDS as part of CME on May 18, 2013.

2. MicroDS can pass through the bow shock and the magnetopause as a magnetized plasmoid. In this case, the velocity of the plasmoid decreases, and the ion con- centration in it increases from $10 \mathrm{~cm}^{-3}$ to $90 \mathrm{~cm}^{-3}$. The correlation coefficient between the SW particle concentration and the geomagnetic field modulus in the magnetized plasmoid passing through the magnetopause into the magnetosphere is negative, as in the microDS in SW.

3 . When the microDS interacts with the magnetopause, a pulsed electric field of $\sim 400 \mathrm{mV} / \mathrm{m}$ is generated with subsequent oscillations with $T \sim 200 \mathrm{~s}$ and an amplitude of $\sim 50 \mathrm{mV} / \mathrm{m}$. The electric field accelerates charged particles of the radiation belt and induces modulated proton fluxes in the energy range $95-575 \mathrm{keV}$ in the dayside magnetosphere and 40-475 keV electron and $95-575 \mathrm{keV}$ proton fluxes on the night side.

4. The SW microDS energy input from the day side causes a magnetospheric disturbance with a maximum intensity $S Y M-H \sim-65 \mathrm{nT}$ and $A E \sim 1220 \mathrm{nT}$ at the initial phase of the moderate magnetic storm (Dst=-61 nT). The increase in auroral magnetic activity at 02:55 UT may be classified as a sawtooth substorm without pronounced growth and expansion phases (breakup). Auroras, modulation of radiation belt particle fluxes, and the sharp increase in the current of the westward electrojet in the form of a local current vortex, similar to that found in [Belakhovsky et al., 2019], are generated by the input of the energy, brought by the microDS into the nightside ionosphere, and by the movement of the microDS from the day side to the magnetotail.

5 . The results presented are consistent with the concept of pulsed penetration of the magnetosheath plasma elements into the magnetosphere, proposed in 1976 [Lemaire, 1977, 1985] and analyzed in [Heikkila, 2003].

The possibility of penetration of a bunch of magnetized plasma into the magnetosphere has been predicted theoretically and may be due to various mechanisms. According to [Lemaire, 1977; Echim, Lemaire, 2000; Voitcu, Echim, 2016], during collision with a bow shock wave in DS, pulsed occurrence of a drift electric field $E$ (this means that $E$ is perpendicular to the magnetic field $B$ and velocity $V$ ), which ensures further motion of DS in the magnetosheath and its penetration through the magnetopause, is possible.

The fact that in the magnetosphere the observed microDS velocity decreases and is significantly lower than the velocity of magnetosonic waves argues for penetration of microDS into the magnetosphere and its motion in it, rather than an MHD wave of any type. The mechanism of pulsed penetration of plasma jets from the magnetosheath into the magnetosphere is confirmed by satellite observations. For example, Gunell et al. [2012] and Dmitriev, Suvorova [2015] have shown that the overwhelming majority of plasma jets penetrating into the magnetosphere feature high velocities $V>220 \mathrm{~km} / \mathrm{s}$ and kinetic pressure $\beta_{\mathrm{k}}>1$, which corresponds to the combination of the finite Larmor radius effect with the impulsive penetration mechanism.

The events depicted in Figure 1 in [Meurant et al., 2005] and in Figure 2 in [Keika et al., 2009] also exemplify DS propagation from the solar wind into the magnetosheath. The structures in SW and magnetosheath displayed in these figures are easily identified with DS by the anticorrelation between the magnetic field $B$ and the 
plasma density $N$. However, the authors call them dynamic pressure jumps $P_{\mathrm{d}}$. They assume that a fast shock wave propagates in the magnetosheath due to the interaction between the dynamic pressure jump $P_{\mathrm{d}}$ and the bow shock wave. A similar case of the interaction is described in [Maynard et al., 2008]. An illustrative example of propagation of an SW diamagnetic structure into the magnetosheath is given in [Rakhmanova et al., 2015]. Large statistics on passage of jets from the magnetosheath to the magnetosphere is also presented in [Dmitriev, Suvorova, 2015 ].

Note that the conclusion about the passage of the diamagnetic structure as a constituent of the solar wind (inhomogeneous region of magnetized plasma) to the magnetosphere and about its movement across geomagnetic field lines differs from the generally accepted point of view on the SW-magnetosphere interaction according to which the dominant mechanism is the reconnection of interplanetary and geomagnetic field lines [Akasofu, 2017].

We express our sincere gratitude to NASA CDAWEB for providing data from ACE, Wind, GOES13, GOES-15, THEMIS satellites. We thank the developers of instruments installed on the satellites and the leaders of the experiments carried out with these satellites for the possibility of using the data. The results reported in this paper are based on data from INTERMAGNET magnetic observatories. We acknowledge the national institutions supporting the observatories [www.intermagnet.org]. The work of Parkhomov V.A. was financially supported by the Ministry of Science and Higher Education of the Russian Federation under the project "Development of a mathematical model of the interaction between solar wind diamagnetic structures and Earth's magnetosphere" and by RFBR (Grant No. 18-55-52006 MNTa). The work of Eselevich V.G. and Eselevich M.G. was performed with budgetary funding of Basic Research program II.16. The work of Tsegmed B. was financially supported by the Academy of Sciences of Mongolia (Grant No. SHUAG_2017/17) and by the Ministry of Education, Science and Sports of Mongolia (project No. SHUSS-2017/65). The work of Khomutov S.Yu. was financially supported by the project "Physical processes in the system of near space and geospheres under solar and lithospheric effects", No. AAAA-A21-121011290003-0. Measurements with induction magnetometers of the observatories Paratunka and Magadan were carried out within the framework of the agreement on academic exchange between IKIR FEB RAS and the Nagoya Research Institute (Japan) sponsored by the PWING project (JSPS KAKENHI 16H06286).

We express our deep gratitude to the leading researcher of IKFIA NP SB RAS Baishev D.G. for help in interpreting the keogram.

\section{REFERENCES}

Akasofu S.-I. Auroral substorms: search for processes causing the expansion phase in terms of the electric current approach. Space Sci Rev. 2017, vol. 212, pp. 341-381. DOI: 10.1007/s11214-017-0363-7.

Belakhovsky V., Pilipenko V., Engebretson M., Sakharov Y., Selivanov V. Impulsive disturbances of the geomagnetic field as a cause of induced currents of electric power lines. Journal of Space Weather and Space Climate. 2019, vol. 9, no. A18, pp. 2-19. DOI: 10.1051/swsc/2019015.

Dmitriev A.V., Suvorova A.V. Large-scale jets in the magnetosheath and plasma penetration across the magnetopause: THEMIS observations. J. Geophys. Res.: Space Phys. 2015, vol. 120, iss. 6. DOI: 10.1002/2014JA020953.

Echim M.M., Lemaire J.F. Laboratory and numerical simulations of the impulsive penetration mechanism. Space Sci. Rev. 2000, vol. 92, pp. 565-601.

Ermolaev Yu.I., Nikolaeva N.S., Lodkina I.G., Ermolaev M.Yu. Catalog of large-scale solar wind phenomena during 1976-2000. Cosmic Res. 2009, vol. 47, no. 2, pp. 81-94. DOI: 10.1134/S0010952509020014.

Eselevich M.V., Eselevich V.G. Sporadic plasma streams and their sources in the period of extraordinary solar activity from October 26 to November 6, 2003. Cosmic Res. 2004, vol. 42, no. 6, pp. 571-582. DOI: 10.1007/s10604-005-0004-x.

Eselevich M.V., Eselevich V.G. Fractal structure of the heliospheric plasma sheet in the Earth's orbit. Geomagnetism and Aeronomy. 2005, vol. 45, no. 3, pp. 326-336.

Gjerloev J.W. The SuperMAG data processing technique, J. Geophys. Res. 2012, vol. 117, A09213. DOI: 10.1029/ 2012JA017683.

Guglielmi A.V. MGD volni v okolozemnoi plazme [MHD Wave in Near Earth Plasma]. Moscow, Nauka Publ, 1979, p. 70. (In Russian).

Gunell H., Nilsson H., Stenberg G., Hamrin M., Karlsson T., Maggiolo R., André M., Lundin R., Dandouras I. Plasma penetration of the dayside magnetopause. Phys. Plasmas. 2012, vol. 19, no. 7, 072906. DOI: 10.1063/1.4739446.

Hess W.N. Radiatsionnyi poyas i magnitosphera [ The Radiation Belts and Magnetosphere]. Moscow, Mir Publ., 1968, 352 p. (In Russian).

Heikkila W.J. Initial condition for plasma transfer events. Earth's Low-Latitude Boundary Layer. Ed. by P.T. Newell, T. Onsager. Washington, AGU, 2003, pp. 157-168. (Geophysical Monograph Ser. Vol. 113). DOI: 10.1029/133gm16.

Hsu T.-S., Tung-Shin, McPherron R.L. An evaluation of the statistical signatures of the association between northward turnings of the interplanetary magnetic field and substorm expansion onset. J. Geophys. Res: Space Phys. 2002, vol. 107, no. A11, 1389. DOI: 10.1029/2000JA000125.

Keika K., Nakamura R., Baumjohann W., Angelopoulos V., Chi P.J., H.Glassmeier K., Fillingim M., Magnes W., Auster H.U., Fornaçon K.H., Reeves G.D., Yumoto K., Lucek E.A., Carr C.M., Dandouras I. Substorm expansion triggered by a sudden impulse front propagating from the dayside magnetopause. $J$. Geophys. Res: Space Phys. 2009, vol. 114, iss. A1. DOI: 10.1029/2008JA013445.

Kessel R.L., Mann I.R., Fung S.F., Milling D.K., O'Connell N. Correlation of Pc5 wave power inside the magnetosphere during high speed streams. Ann. Geophys. 2004, vol. 22, pp. 629-641. DOI: 10.5194/angeo-22-629-2004.

Klibanova Yu.Yu., Mishin V.V. Tsegmed B., Moiseev A.V. Properties of Daytime Long-Period Pulsations during Magnetospheric Storm Commencement. ISSN 0016-7932, Geomagnetism and Aeronomy. 2016, vol. 56, no. 4, pp. 426-440. DOI: 10.1134/S0016793216040071.

Kokubun S., McPherron R., Russel C. Triggering of Substorms by Solar Wind Discontinuities. J. Geophys. Res. 1977, vol. 82, no. 1, pp. 74-86. DOI: 10.1029/JA082i001p00074.

Koskinen H.E.J., Lopez R.E., Pulkkinen R.I., Baker D.N., Bosinger T. Pseudobreakup and substorm growth phase in the ionosphere and magnetosphere. J. Geophys. Res. 1993. vol. 98, p. 5801-5814.DOI: 10.1029/92JA02482.

Lemaire J. Impulsive penetration of filamentary plasma elements into themagnetospheres of the Earth and Jupiter. Planet. Space Sci. 1977, vol. 25, pp. 887-890. DOI: 10.1016/00320633(77)90042-3. 
Lemaire J. Plasmoid motion across a tangential discontinuity (with application to the magnetopause). J. Plasma Phys. 1985, vol. 33, no. 3, pp. 425-436.

Liou K., Newell P.T., Meng C.-I., Wu C.-C., Lepping P. Investigation of external triggering of substorms with Polar ultraviolet imager observations. J. Geophys. Res. 2003, vol. 108, no. A10, 1364. DOI: 10.1029/2003JA009984.

Maynard N.C., Farrugia C.J., Ober D.M., Burke W.J., Dunlop M., Mozer F.S., et al. Cluster observations of fast shocks in the magnetosheath launched as a tangential discontinuity with a pressure increase crossed the bow shock. J. Geophys. Res. 2008, vol. 113, A10212. DOI: 10.1029/2008JA013121.

Meurant M., Gerard J.C., Blockx C., Coumans V., Hubert B., Connors M., Lyons L.R., Donovan E. Comparison of intense nightside shock-induced precipitation and substorm activity. $J$. Geophys. Res. 2005, vol. 110, A07228. DOI: 10.1029/2004JA 010916.

Mishin V.V. On the wave energy flux to the magnetosphere under the action of solar wind pressure pulsations. Issledovaniya po geomagnetizmu, aeronomii i fizike Solntsa [Research on Geomagnetism, Aeronomy and Solar Physics]. Novosibirsk, Nauka Publ., 1996, iss. 104, pp. 182-185. (In Russian).

Mishin V.V., Tsegmed B., Klibanova Y.Y., Kurikalova M.A. Burst geomagnetic pulsations as indicators of substorm expansion onsets during storms. J. Geophys. Res.: Space Phys. 2020, vol. 125, iss. 10, e2020JA028521. DOI: 10.1029/2020JA028521.

Parkhomov V.A., Borodkova N.L., Eselevich V.G., Eselevich M.V., Dmitriev A.V., Chilikin V.E. Features of the impact of the solar wind diamagnetic structure on Earth's magnetosphere. Solar-Terr. Phys. 2017, vol. 3, iss. 4, pp.4457. DOI: $10.12737 /$ stp-34201705.

Parkhomov V.A., Borodkova N.L., Eselevich V.G., Eselevich M.V., Dmitriev A.V., Chilikin V.E. Solar wind diamagnetic structures as a source of substorm - like disturbances. J. Atmos. Solar-Terr. Phys. 2018, vol. 181, pp.55-67. DOI: $10.1016 /$ j.jastp.2018.10.010.

Parkhomov V.A., Eselevich V.G., Eselevich M.V, Dmitriev A.V., Suvorova A.V., Vedernikova T.I. Classification of magnetospheric responses to interaction with diamagnetic structures of slow solar wind. Solar-Terr. Phys. 2020, vol. 6, iss. 4, pp. 24-36. DOI: 10.12737/stp-64202004.

Potapov A.S., Polyushkina T.N. Experimental evidence for direct penetration of ULF waves from the solar wind and their possible effect on acceleration of radiation belt electrons. Geomagnetism and Aeronomy. 2010, vol. 50, no. 8, pp. 950-957. DOI: $10.1134 /$ S0016793210080049.

Rakhmanova L.S., Riazantseva M.O., Zastenker G.N. Solar-wind structure propagation through the magnetosheath studied by two Themis probes. Cosmic Res. 2015, vol. 53, no.
5, pp. 331-340. DOI: 10.1134/S0010952515040073.

Schwenn R., Dal Lago A., Huttunen E., Gonzalez W.D. The association of coronal mass ejections with their effects near the Earth. Ann. Geophys. 2005, vol. 23, pp. 1033-1059. DOI: 10.5194/angeo-23-1033-2005.

Shadrina L.P., Starodubtsev S.A. Manifestation of interplanetary shock in the geomagnetic storms and substorms. Physics of Auroral Phenomena. Proc. XXXIX Annual Seminar. Apatity, 2016, pp. 23-26. DOI: 10.22323/1.236.0111.

Troshichev O.A., Stauning P., Liou K., Reeves G. Sawtooth substorms: Inconsistency of repetitive bay-like magnetic disturbances with behavior of aurora. Adv. Space Res. 2011, vol. 47, pp. 702-709. DOI: 10.1016/j.asr.2010.09.026.

Voitcu G., Echim M. Transport and entry of plasma clouds/jets across transverse magnetic discontinuities: threedimensional electromagnetic particle-in-cell simulations. $J$. Geophys. Res.: Space Phys. 2016, vol. 121, pp. 4343-4361. DOI: 10.1002/2015JA021973.

Yahnin A.G., Sergeev V.A., Bösinger T., Sergienko T.I., Kornilov I.A., Borodkova N.L., Thomsen M.F., Belian R.D., Sauvaud J.-A., Lutsenko V.N., Skalsky A.A. Correlated Interball/ground-based observations of isolated substorm. Ann. Geophys. 2001, vol. 19, pp. 687-698. DOI: 10.5194/angeo-19687-2001.

URL: https://cdaw.gsfc.nasa.gov/CME_list/catalog description.htm (accessed November 20, 2020).

URL: https://satdat.ngdc.noaa.gov/sem/goes/data/new_avg (accessed November 20, 2020).

URL: http://cdaweb.gsfc.nasa.gov/cgi-bin/eval2.cgi (accessed November 20, 2020).

URL: http://www.obsebre.es/en/rapid (accessed November 20, 2020).

URL: http://supermag.jhuapl.edu/mag (accessed November 20, 2020).

URL: http://carisma.ca/carisma-data/fgm-pi2-dbase (accessed November 20, 2020). 2020).

URL: www.intermagnet.org (accessed November 20,

URL: https://cdaweb.sci.gsfc.nasa.gov/cdaweb/istp_public (accessed November 20, 2020).

URL: https://www.sgo.fi/Data/Riometer/rioData.php (accessed November 20, 2020).

How to cite this article

Parkhomov V.A., Eselevich V.G., Eselevich M.V., Dmitriev A.V., Suvorova A.V., Khomutov S.Yu., Tsegmed B., Raita T. Magnetospheric response to the interaction with the sporadic solar wind diamagnetic structure. Solar-Terrestrial Physics. 2021. Vol. 7. Iss. 3. P. 11-28. DOI: $10.12737 /$ stp-73202102. 